\title{
Globally Convergent Adaptive Tracking of Angular Velocity and Inertia Identification for a 3-DOF Rigid Body
}

\author{
Nalin A. Chaturvedi, Dennis S. Bernstein, Fellow, IEEE, Jasim Ahmed, Fabio Bacconi, and
} N. Harris McClamroch, Fellow, IEEE

\begin{abstract}
This paper addresses the problem of a rigid body, with unknown inertia matrix, tracking a desired angular velocity reference using adaptive feedback control. The control law, which has the form of a sixth-order dynamic compensator, does not require knowledge of the inertia of the rigid body. A Lyapunov argument is used to guarantee that asymptotic tracking is achieved globally. Furthermore, an analytical expression for an upper bound on the magnitude of the required torque is presented for a given reference signal. Next, sufficient conditions on the reference signal are given under which asymptotic identification of the inertia matrix is achieved. Reference signals that satisfy these sufficient conditions are characterized and simulation results that illustrate the control algorithm are presented for a constant spin about a fixed axis and for sinusoidal spins about the body axes. The controller is implemented on an experimental testbed, and experiments are performed for several commanded reference signals. The experimental results demonstrate the tracking performance of the controller, and parameter convergence is observed.
\end{abstract}

Index Terms-Adaptive control, angular velocity tracking, control saturation, estimation, inertia identification, rotating bodies, space vehicle control.

\section{INTRODUCTION}

TABILIZATION of a single free rigid body in three dimensions is a widely studied and a fundamental problem in spacecraft dynamics. Although the problem is trivial in the presence of three control torques, significant research has been devoted to the cases of two torques [2]-[10] and one torque [3], [4], [11]. If minimum fuel or miminum time performance is required in addition to stabilization, then this problem is challenging even in the case of three torques [12]-[14]. When rotors are used to provide control torques, the problem involves multiple bodies and significantly greater complexity [15], [16].

The above discussion is based on the assumption that the spacecraft mass distribution is known. In practice, however, fuel usage, moving appendages, and complex geometry limit the ability to determine the mass distribution with arbitrary accuracy. Hence, it is of interest to determine stabilizing controllers that can operate with minimal inertia information. This moti-

Manuscript received January 05, 2005. Manuscript received in final form April 5, 2006. Recommended by Associate Editor D. W. Repperger. This work was supported in part by the Air Force Office of Scientific Research under Grant F49620-98-1-0037 and by the National Science Foundation under Grants ECS-0140053 and ECS-0244977.

The authors are with the Department of Aerospace Engineering, University of Michigan, Ann Arbor, MI 48109 USA (e-mail: dsbaero@umich.edu).

Digital Object Identifier 10.1109/TCST.2006.876908 vates the use of Lyapunov-based techniques to design an adaptive controller [17].

In the present paper, we address the inertia uncertainty problem by deriving an adaptive controller that tracks an angular velocity reference without any information concerning the mass distribution. In addition, we present sufficient conditions on the reference signal that guarantee asymptotic identification of the inertia matrix of the rigid body. The reference signal need not be periodic. Reference signals that satisfy these sufficient conditions include a constant spin about a body-fixed axis and a sinusoidal spin about a body axis. The controller provides asymptotic tracking of angular velocity reference signals. For a rotating spacecraft modeled as a rigid body, the adaptive tracking controller is effectively a PI control law involving six integrators whose values correspond to estimates of the entries of the inertia matrix. The case of single degree-of-freedom (DOF) rotation with an input nonlinearity was considered in [1].

It is important to point out that angular velocity tracking does not imply attitude tracking. Attitude control of a spacecraft under inertia matrix uncertainty was studied in [18]. The inclusion of attitude states within an inertia-independent adaptive controller is given in [19]. The tracking problem, considered in the present paper, can be viewed as an extension of [19] to the case in which attitude measurements are not available. Furthermore, we present sufficient conditions on the reference signal that guarantee asymptotic identification of the unknown inertia. Also, the present paper includes experimental results obtained from implementing the controller on an air-bearing testbed. These results demonstrate the tracking performance and parameter identification ability of the controller.

The contents of the paper are as follows. In Section II, we consider the three-dimensional (3-D) case of a rotating rigid body. We develop the equations of motion for a rotating rigid body, and we rewrite these equations in a form that isolates the uncertain inertia parameters. Next, in Section III, we present an adaptive tracking control law that requires no knowledge of the inertia matrix. In Sections IV and V, we present methods for identifying the inertia matrix with aperiodic and periodic reference signals, respectively. Simulations and numerical examples are presented in Section VI to illustrate the tracking and identification algorithms. In Section VII, we discuss implementation issues for the control algorithm, and we provide details of an experimental testbed (TACT). In Section VIII, we discuss experimental results obtained from the implementation of the algorithm. Finally, we close with conclusions in Section IX. 


\section{Rigid Body EQuations of Motion}

Consider a rigid body, such as a spacecraft, with actuators that provide body-fixed torques about three mutually perpendicular axes that define a body-fixed frame $\mathcal{B}$ located at the center of mass of the body. For each axis a body-fixed torque can be obtained by employing, for example, a pair of actuators to produce equal and opposite forces perpendicular to the line joining the actuators. The lines joining each pair of actuators need not pass through the center of mass.

For $t \geq 0$, the angular velocity $\omega=\omega(t) \in \mathbb{R}^{3}$ of $\mathcal{B}$ with respect to an inertial frame $I$, and resolved in $\mathcal{B}$, satisfies

$$
\dot{\omega}=-J^{-1} \omega^{\times} J \omega+J^{-1} \tau
$$

where

$$
J=\left[\begin{array}{lll}
J_{11} & J_{12} & J_{13} \\
J_{12} & J_{22} & J_{23} \\
J_{13} & J_{23} & J_{33}
\end{array}\right]
$$

is the constant positive-definite inertia matrix of the body, also resolved in $\mathcal{B}$, and $\tau=\tau(t) \in \mathbb{R}^{3}$ is the vector of control torques. The notation $a^{\times}$for $a=\left[a_{1} a_{2} a_{3}\right]^{\mathrm{T}}$ denotes the skewsymmetric matrix

$$
\left[\begin{array}{ccc}
0 & -a_{3} & a_{2} \\
a_{3} & 0 & -a_{1} \\
-a_{2} & a_{1} & 0
\end{array}\right]
$$

The inertia matrix $J$ is assumed to be unknown.

Let $\nu:[0, \infty) \mapsto \mathbb{R}^{3}$ denote the desired angular velocity of $\mathcal{B}$ with respect to $I$. We assume that $\nu$ is differentiable. Defining the angular velocity error

$$
\tilde{\omega} \triangleq \omega-\nu
$$

it follows from (1) that $\tilde{\omega}$ satisfies

$$
\dot{\tilde{\omega}}=-J^{-1}(\tilde{\omega}+\nu)^{\times} J(\tilde{\omega}+\nu)-\dot{\nu}+J^{-1} \tau .
$$

The control objective is to determine $\tau$ such that $\tilde{\omega}(t) \rightarrow 0$ as $t \rightarrow \infty$ for all initial conditions $\omega(0)$ and without knowledge of $J$.

\section{AdAPTIVE CONTROL LAW}

In this section, we present a feedback control law that asymptotically tracks the reference angular velocity $\nu(t)$. We define a

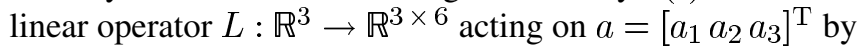

$$
L(a) \triangleq\left[\begin{array}{cccccc}
a_{1} & 0 & 0 & 0 & a_{3} & a_{2} \\
0 & a_{2} & 0 & a_{3} & 0 & a_{1} \\
0 & 0 & a_{3} & a_{2} & a_{1} & 0
\end{array}\right]
$$

Letting

$$
\mathcal{J} \triangleq\left[J_{11} J_{22} J_{33} J_{23} J_{13} J_{12}\right]^{\mathrm{T}}
$$

it follows that:

$$
J a=L(a) \mathcal{J} .
$$

Equation (3) can now be rewritten in the form

$$
\dot{\tilde{\omega}}=-J^{-1} F(\tilde{\omega}, \nu, \dot{\nu}) \mathcal{J}+J^{-1} \tau
$$

where $F: \mathbb{R}^{3} \times \mathbb{R}^{3} \times \mathbb{R}^{3} \rightarrow \mathbb{R}^{3 \times 6}$ is defined by

$$
F(\tilde{\omega}, \nu, \dot{\nu}) \triangleq(\tilde{\omega}+\nu)^{\times} L(\tilde{\omega}+\nu)+L(\dot{\nu}) .
$$

We now present an adaptive controller for angular velocity tracking based on an estimate $\hat{\mathcal{J}}$ of $\mathcal{J}$. We denote the inertia estimate error by $\tilde{\mathcal{J}} \triangleq \hat{\mathcal{J}}-\mathcal{J}$. Furthermore, let $\|\cdot\|_{2}$ denote the Euclidean norm.

Theorem 1: Assume that $\nu$ is differentiable and bounded, and that $\dot{\nu}$ is piecewise continuous and bounded. Let $K \in \mathbb{R}^{3 \times 3}$ and $Q \in \mathbb{R}^{6 \times 6}$ be positive definite, and consider the closed-loop system consisting of (5) and the adaptation and control law

$$
\begin{aligned}
\dot{\hat{\mathcal{J}}} & =-Q F^{\mathrm{T}}(\tilde{\omega}, \nu, \dot{\nu}) \tilde{\omega} \\
\tau & =-K \tilde{\omega}+F(\tilde{\omega}, \nu, \dot{\nu}) \hat{\mathcal{J}} .
\end{aligned}
$$

Then, the zero solution of the closed-loop dynamics of (5), (7), and (8) given in error coordinates by

$$
\begin{aligned}
& \dot{\tilde{\omega}}=-J^{-1} K \tilde{\omega}+J^{-1} F(\tilde{\omega}, \nu, \dot{\nu}) \tilde{\mathcal{J}} \\
& \tilde{\mathcal{J}}=-Q F^{\mathrm{T}}(\tilde{\omega}, \nu, \dot{\nu}) \tilde{\omega}
\end{aligned}
$$

is Lyapunov stable. Furthermore, for all $\omega(0) \in \mathbb{R}^{3}$ and $\hat{\mathcal{J}}(0) \in \mathbb{R}^{6}$, the solution of the closed-loop system (9), (10) satisfies $\lim _{t \rightarrow \infty} \tilde{\omega}(t)=0, \lim _{t \rightarrow \infty} \tilde{\mathcal{J}}(t)=0$, and $\lim _{t \rightarrow \infty}\left\|Q^{-1 / 2} \tilde{\mathcal{J}}(t)\right\|_{2}$ exists.

Proof: Equations (9) and (10), comprise a nine-dimensional (9-D) nonlinear, time-varying system, for which $[\tilde{\omega} \tau \tilde{\mathcal{J}} \tau]=\left[\begin{array}{ll}0 & 0\end{array}\right]$ is an equilibrium. Furthermore, since $\nu$ is bounded and $\dot{\nu}$ is piecewise continuous and bounded, the right-hand side of (9) and (10) is piecewise continuous in time and locally Lipschitz in states, uniformly in time. Thus, solutions of the closed-loop system (9), (10) exist.

To prove asymptotic tracking, consider the positive-definite Lyapunov candidate

$$
V(\tilde{\omega}, \tilde{\mathcal{J}})=\frac{1}{2}\left(\tilde{\omega}^{\mathrm{T}} J \tilde{\omega}+\tilde{\mathcal{J}}^{\mathrm{T}} Q^{-1} \tilde{\mathcal{J}}\right)
$$


which does not explicitly depend on time and is radially unbounded. The total time derivative of $V$, along trajectories of the closed-loop system, is given by

$$
\begin{aligned}
\dot{V}(\tilde{\omega}, \tilde{\mathcal{J}}) & =-\tilde{\omega}^{\mathrm{T}} K \tilde{\omega}+\tilde{\mathcal{J}}^{\mathrm{T}}\left[F^{\mathrm{T}}(\sigma, \nu, \dot{\nu}) \tilde{\omega}+Q^{-1} \dot{\mathcal{J}}\right] \\
& =-\tilde{\omega}^{\mathrm{T}} K \tilde{\omega}
\end{aligned}
$$

which shows that $\dot{V}$ is negative semidefinite and is not an explicit function of time. Hence, Theorem 8.4 of [20] implies that for all initial conditions $\tilde{\omega}(0)$ and $\tilde{\mathcal{J}}(0)$, the solutions of (9) and (10) are bounded and approach the set $E \triangleq \dot{V}^{-1}(0)=$ $\left\{[\tilde{\omega} \tilde{\mathcal{J}}] \in \mathbb{R}^{9}: \tilde{\omega}=0\right\}$. Hence, $\tilde{\omega}(t) \rightarrow 0$ as $t \rightarrow \infty$. Also, since $V(\cdot)$ is positive definite and $\dot{V}(\cdot)$ is negative semidefinite, it follows that the equilibrium $(0,0)$ of the system (9) and (10) is Lyapunov stable.

Since $\tilde{\omega}(t) \rightarrow 0$ as $t \rightarrow \infty$ and $\nu$ and $\dot{\nu}$ are bounded, it follows from (10) that $\dot{\mathcal{J}}(t) \rightarrow 0$ as $t \rightarrow \infty$. Furthermore, $\tilde{\omega}(t)$ and $\tilde{\mathcal{J}}(t)$, and, hence, $\hat{\mathcal{J}}(t)$, are bounded. Now, since $\dot{V}(t) \triangleq$ $\dot{V}(\tilde{\omega}(t), \tilde{\mathcal{J}}(t)) \leq 0$ and $V(t) \triangleq V(\tilde{\omega}(t), \tilde{\mathcal{J}}(t)) \geq 0$ for all $t \geq 0$, it follows that $\lim _{t \rightarrow \infty} V(t)$ exists.

Next, letting $t \rightarrow \infty$ in (11) yields

$$
\lim _{t \rightarrow \infty} \tilde{\mathcal{J}}^{\mathrm{T}}(t) Q^{-1} \tilde{\mathcal{J}}(t)=2 \lim _{t \rightarrow \infty} V(t)
$$

where the right-hand side exists. Hence, $\lim _{t \rightarrow \infty}\left\|Q^{-1 / 2} \tilde{\mathcal{J}}(t)\right\|_{2}$ exists.

\section{INERTIA IDENTIFICATION}

Note that the control law (7) and (8) does not require knowledge of the inertia $\mathcal{J}$. Although $\dot{\mathcal{J}}$ converges to zero and $\left\|Q^{-1 / 2} \hat{\mathcal{J}}\right\|_{2}$ converges, $\hat{\mathcal{J}}$ does not necessarily converge and, even if $\hat{\mathcal{J}}$ converges, it does not necessarily converge to the actual inertia $\mathcal{J}$. We now give sufficient conditions under which $\hat{\mathcal{J}}$ converges to $\mathcal{J}$. The following lemmas and notation are needed. Define $W: \mathbb{R}^{3} \times \mathbb{R}^{3} \rightarrow \mathbb{R}^{3 \times 6}$ by

$$
W(\nu, \dot{\nu}) \triangleq F(0, \nu, \dot{\nu})=\nu^{\times} L(\nu)+L(\dot{\nu}) .
$$

Lemma 1: Consider the closed-loop system (9) and (10) under the assumptions of Theorem 1 . Then

$\lim _{t \rightarrow \infty} W(\nu(t), \dot{\nu}(t)) \tilde{\mathcal{J}}(t)=\lim _{t \rightarrow \infty} F(\tilde{\omega}(t), \nu(t), \dot{\nu}(t)) \tilde{\mathcal{J}}(t)=0$

Proof: Theorem 1 implies that $\tilde{\omega}(t) \rightarrow 0$ as $t \rightarrow \infty$. Then

$$
\lim _{t \rightarrow \infty} \int_{0}^{t} \dot{\tilde{\omega}}(s) d s=\lim _{t \rightarrow \infty} \tilde{\omega}(t)-\tilde{\omega}(0)=-\tilde{\omega}(0) .
$$

Since $\nu$ is continuous and bounded and $\dot{\nu}$ is piecewise continuous and bounded, (9) implies that $\dot{\tilde{\omega}}(t)$ is globally piecewise uniformly continuous (see [1] for definition). Now, applying the generalized version of Barbalat's Lemma given in Appendix B of [1] yields $\lim _{t \rightarrow \infty} \dot{\tilde{\omega}}(t)=0$. Therefore, it follows from (9) that

$$
\lim _{t \rightarrow \infty} F(\tilde{\omega}(t), \nu(t), \dot{\nu}(t)) \tilde{\mathcal{J}}(t)=0 .
$$

Note that

$$
\begin{aligned}
& {[F(\tilde{\omega}(t), \nu(t), \dot{\nu}(t))-W(\nu(t), \dot{\nu}(t))] \tilde{\mathcal{J}}(t)} \\
& \quad=\left[\tilde{\omega}(t)^{\times} L(\tilde{\omega}(t))+\tilde{\omega}(t)^{\times} L(\nu(t))+\nu(t)^{\times} L(\tilde{\omega}(t))\right] \tilde{\mathcal{J}}(t) .
\end{aligned}
$$

Since $\tilde{\omega}(t) \rightarrow 0$ as $t \rightarrow \infty$ and $\tilde{\mathcal{J}}(t)$ and $\nu(t)$ are bounded, (17) implies that

$$
\lim _{t \rightarrow \infty}[F(\tilde{\omega}(t), \nu(t), \dot{\nu}(t))-W(\nu(t), \dot{\nu}(t))] \tilde{\mathcal{J}}(t)=0 .
$$

Furthermore, since

$$
\begin{aligned}
W(\nu(t), \dot{\nu}(t)) \tilde{\mathcal{J}}(t) & =F(\tilde{\omega}(t), \nu(t), \dot{\nu}(t)) \tilde{\mathcal{J}}(t) \\
& -[F(\tilde{\omega}(t), \nu(t), \dot{\nu}(t))-W(\nu(t), \dot{\nu}(t))] \tilde{\mathcal{J}}(t)
\end{aligned}
$$

it follows from (16) and (18) that

$$
\lim _{t \rightarrow \infty} W(\nu(t), \dot{\nu}(t)) \tilde{\mathcal{J}}(t)=0
$$

For $H \in \mathbb{R}^{m \times n}$, denote the $i$ th largest singular value of $H$ by $\sigma_{i}(H)$. Furthermore, $\sigma_{\max }(H) \triangleq \sigma_{1}(H)$ and $\sigma_{\min }(H) \triangleq \sigma_{\min \{m, n\}}(H)$ denote the largest and the smallest singular values of $H$, respectively.

Lemma 2: Consider the closed-loop system (9), (10), under the assumptions of Theorem 1. Furthermore, for $t \geq 0$, define $W(t) \triangleq W(\nu(t), \dot{\nu}(t))$. Then, for every $\varepsilon>0$ and every $\delta>0$, there exists $T \geq 0$ such that, for all $t_{0} \geq T$ and $\bar{t}_{0} \in\left[t_{0}, t_{0}+\delta\right]$, $\left\|W\left(t_{0}\right) \tilde{\mathcal{J}}\left(\bar{t}_{0}\right)\right\|_{2}<\varepsilon$.

Proof: Let $\varepsilon>0$ and $\delta>0$. Since $\nu(t)$ and $\dot{\nu}(t)$ are bounded for all $t \geq 0$, it follows from the definition of $W(t)$ and (13) that $W(t)$ is bounded for all $t \geq 0$ and, hence, $\sigma_{\max } W(t)$ is bounded for all $t \geq 0$. Define $\bar{\sigma} \triangleq \max _{t \geq 0} \sigma_{\max } W(t)$ and choose $\varepsilon_{1}=(\varepsilon / 2)$ and $\varepsilon_{2}=(\varepsilon / 2 \bar{\sigma} \delta)$.

Next, it follows from (14) that there exists $T_{1} \geq 0$ such that, for every $t \geq T_{1},\|W(t) \tilde{\mathcal{J}}(t)\|_{2}<\varepsilon_{1}$. Similarly, since $\dot{\mathcal{J}}(t) \rightarrow$ 0 as $t \rightarrow \infty$, it follows that there exists a $T_{2} \geq 0$, such that for every $t \geq T_{2},\|\tilde{\mathcal{J}}\|_{2}<\varepsilon_{2}$. Choose $T=\max \left(T_{1}, T_{2}\right)$. Then, for every $t_{0} \geq T$ and $\bar{t}_{0} \in\left[t_{0}, t_{0}+\delta\right]$

$$
\begin{aligned}
& \left\|W\left(t_{0}\right) \tilde{\mathcal{J}}\left(\bar{t}_{0}\right)\right\|_{2} \\
& \quad=\left\|W\left(t_{0}\right) \tilde{\mathcal{J}}\left(\bar{t}_{0}\right)+W\left(t_{0}\right)\left(\tilde{\mathcal{J}}\left(\bar{t}_{0}\right)-\tilde{\mathcal{J}}\left(t_{0}\right)\right)\right\|_{2} \\
& \quad \leq\left\|W\left(t_{0}\right) \tilde{\mathcal{J}}\left(t_{0}\right)\right\|_{2}+\left\|W\left(t_{0}\right)\left[\tilde{\mathcal{J}}\left(\bar{t}_{0}\right)-\tilde{\mathcal{J}}\left(t_{0}\right)\right]\right\|_{2} \\
& \quad \leq\left\|W\left(t_{0}\right) \tilde{\mathcal{J}}\left(t_{0}\right)\right\|_{2}+\bar{\sigma}\left\|\tilde{\mathcal{J}}\left(\bar{t}_{0}\right)-\tilde{\mathcal{J}}\left(t_{0}\right)\right\|_{2} \\
& \quad \leq\left\|W\left(t_{0}\right) \tilde{\mathcal{J}}\left(t_{0}\right)\right\|_{2}+\bar{\sigma} \int_{t_{0}}^{\bar{t}_{0}}\|\tilde{\tilde{\mathcal{J}}}(\tau)\|_{2} d \tau \\
& \quad<\varepsilon_{1}+\bar{\sigma} \varepsilon_{2} \delta=\varepsilon .
\end{aligned}
$$


Define $\mathcal{W}:[0, \infty)^{6} \rightarrow \mathbb{R}^{18 \times 6}$ by

$$
\mathcal{W}\left(t_{1}, \ldots, t_{6}\right) \triangleq\left[\begin{array}{c}
W\left(t_{1}\right) \\
\vdots \\
W\left(t_{6}\right)
\end{array}\right]
$$

where $W(\cdot)$ is defined in Lemma 2.

Lemma 3: Consider the closed-loop system (9) and (10) under the assumptions of Theorem 1. Then, for every $\varepsilon>0$ and $\delta>0$, there exists $\bar{T} \geq 0$ such that, for every $T \geq \bar{T}$ and $t_{1}, \ldots, t_{6} \in[T, T+\delta],\left\|\mathcal{W}\left(t_{1}, \ldots, t_{6}\right) \tilde{\mathcal{J}}(T+\delta)\right\|_{2}<\varepsilon$.

Proof: Let $\varepsilon>0$ and $\delta>0$. Then, Lemma 2 implies that there exist $\bar{T} \geq 0$, such that, for every $t_{0} \geq \bar{T}$, $\mid W\left(t_{0}\right) \tilde{\mathcal{J}}\left(\bar{t}_{0}\right) \|_{2}<(\varepsilon / \sqrt{6})$, where $\bar{t}_{0} \in\left[t_{0}, t_{0}+\delta\right]$. Consider any $T \geq \bar{T}$ and $t_{i} \in[T, T+\delta]$, where $i=1, \ldots, 6$. Then, note that $(T+\delta) \in\left[t_{i}, t_{i}+\delta\right], i=1, \ldots, 6$. Hence, substituting $t_{0}=t_{i}$ and $\bar{t}_{0}=T+\delta$, yields $\left\|W\left(t_{i}\right) \tilde{\mathcal{J}}(T+\delta)\right\|_{2}^{2}<\left(\varepsilon^{2} / 6\right), i=1, \ldots, 6$. Thus

$$
\begin{aligned}
\|\mathcal{W} \tilde{\mathcal{J}}(T+\delta)\|_{2}^{2}= & \left\|W\left(t_{1}\right) \tilde{\mathcal{J}}(T+\delta)\right\|_{2}^{2}+\ldots \\
& +\left\|W\left(t_{6}\right) \tilde{\mathcal{J}}(T+\delta)\right\|_{2}^{2} \\
< & \frac{\varepsilon^{2}}{6}+\ldots+\frac{\varepsilon^{2}}{6}=\varepsilon^{2} .
\end{aligned}
$$

The next result provides a sufficient condition for convergence of the inertia estimate $\hat{\mathcal{J}}$ to the actual inertia $\mathcal{J}$.

Theorem 2: Consider the closed-loop system consisting of (5) and the adaptive control law (7) and (8), where $K$ and $Q$ are positive definite. Suppose there exist $\delta>0$ and $\gamma>0$ such that, for every $T \geq 0$, there exist $t_{1}, \ldots, t_{6} \in[T, T+\delta]$ such that $\sigma_{\min }\left(\mathcal{W}\left(t_{1}, \ldots, t_{6}\right)\right)>\gamma$. Then $\lim _{t \rightarrow \infty} \hat{\mathcal{J}}(t)=\mathcal{J}$.

Proof: Theorem 1 implies that $\lambda \triangleq \lim _{t \rightarrow \infty}\left\|Q^{-1 / 2} \tilde{\mathcal{J}}(t)\right\|_{2}$ exists. If $\lambda=0$, then, since $Q^{-1 / 2}$ is nonsingular, $\lim _{t \rightarrow \infty} \tilde{\mathcal{J}}(t)=0$. Now suppose $\lambda \neq 0$. Then, for every $\varepsilon_{1}>0$, there exists $T_{1} \geq 0$ such that, for every $t \geq T_{1},\left|\lambda-\left\|Q^{-1 / 2} \tilde{\mathcal{J}}(t)\right\|_{2}\right|<\varepsilon_{1}$. Choose $\varepsilon_{1}<\lambda / 2$. Hence, for every $t \geq T_{1},\left\|Q^{-1 / 2} \tilde{\mathcal{J}}(t)\right\|_{2}>\lambda / 2$. Furthermore, $\left\|Q^{-1 / 2} \tilde{\mathcal{J}}(t)\right\|_{2} \leq \sigma_{\max }\left(Q^{-1 / 2}\right)\|\tilde{\mathcal{J}}(t)\|_{2}$. Hence, for every $t \geq T_{1},\|\tilde{J}(t)\|_{2}>(1 / 2) \lambda / \sigma_{\max }\left(Q^{-1 / 2}\right)$.

Next, by assumption, for every $T \geq T_{1}$, there exist $t_{1}, \ldots, t_{6} \in[T, T+\delta]$, such that $\sigma_{\min }\left(\mathcal{\mathcal { W }}\left(t_{1}, \ldots, t_{6}\right)\right)>\gamma$. Therefore, it follows that, for every $T \geq T_{1}$, there exist $t_{1}, \ldots, t_{6} \in[T, T+\delta]$ such that

$$
\begin{aligned}
\left\|\mathcal{W}\left(t_{1}, \ldots, t_{6}\right) \tilde{\mathcal{J}}(T+\delta)\right\|_{2} & \geq \sigma_{\min }\left(\mathcal{W}\left(t_{1}, \ldots, t_{6}\right)\right)\|\tilde{\mathcal{J}}(T+\delta)\|_{2} \\
& >\frac{\gamma}{2} \lambda / \sigma_{\max }\left(Q^{-1 / 2}\right) .
\end{aligned}
$$

However, Lemma 3 implies that there exists $T_{2} \geq 0$ such that, for every $T_{3} \geq T_{2}$ and $t_{1}, \ldots, t_{6} \in\left[T_{3}, T_{3}+\right.$ $\delta],\left\|\mathcal{W}\left(t_{1}, \ldots, t_{6}\right) \tilde{\mathcal{J}}\left(T_{3}+\delta\right)\right\|_{2}<(\gamma / 2) \lambda / \sigma_{\max }\left(Q^{-1 / 2}\right)$, which is a contradiction. Hence, $\lambda=0$, and thus, $\lim _{t \rightarrow \infty} \hat{\mathcal{J}}(t)=\mathcal{J}$.

Next, we provide an analytical expression for an upper bound on the maximal magnitude of the torque required to track a given reference signal. To compute this bound, we require knowledge of an upper bound on the initial angular velocity error and the initial inertia estimate error. We denote these bounds by $m_{1}$ and $m_{2}$, respectively. Thus, let $m_{1} \geq 0$ and $m_{2} \geq 0$ satisfy $\|\tilde{\omega}(0)\|_{2} \leq m_{1}$ and $\|\tilde{\mathcal{J}}(0)\|_{2} \leq m_{2}$.

In Theorem 1, it was assumed that $\nu(t)$ and $\dot{\nu}(t)$ are bounded in $[0, \infty)$. Let $\eta_{1} \geq 0$ and $\eta_{2} \geq 0$ satisfy $\|\nu(t)\|_{2} \leq \eta_{1}$, and $\|\dot{\nu}(t)\|_{2} \leq \eta_{2}$ for all $t \geq 0$. Furthermore, let $m_{\mathcal{J}}, \sigma_{J}^{\text {sup }}$ and $\sigma_{J}^{\text {inf }}$ be positive numbers satisfying $\|\mathcal{J}\|_{2} \leq m_{\mathcal{J}}$ and $\sigma_{J}^{\text {sup }} \geq$ $\sigma_{\max }(J) \geq \sigma_{\min }(J) \geq \sigma_{J}^{\text {inf }}$.

Theorem 3: Consider the closed-loop system consisting of (5) and the adaptive control law (7) and (8), where $K$ and $Q$ are positive definite. Denote

$M \triangleq \sigma_{\max }(K) \bar{m}_{1}+\sqrt{6}\left(\bar{m}_{1}+\eta_{1}\right)^{2}\left(\bar{m}_{2}+m_{\mathcal{J}}\right)+\sqrt{6}\left(\bar{m}_{2}+m_{\mathcal{J}}\right) \eta_{2}$

where

$$
\begin{aligned}
& \bar{m}_{1} \triangleq \sqrt{\frac{\sigma_{J}^{\text {sup }}}{\sigma_{J}^{\text {inf }}} m_{1}^{2}+\frac{m_{2}^{2}}{\sigma_{\min }(Q) \sigma_{J}^{\text {inf }}}} \\
& \bar{m}_{2} \triangleq \sqrt{\sigma_{J}^{\sup } \sigma_{\max }(Q) m_{1}^{2}+\frac{\sigma_{\max }(Q)}{\sigma_{\min }(Q)} m_{2}^{2}} .
\end{aligned}
$$

Then, for all $t \geq 0,\|\tilde{\omega}(t)\|_{2} \leq \bar{m}_{1},\|\tilde{\mathcal{J}}(t)\|_{2} \leq \bar{m}_{2}$ and $\|\tau(t)\|_{2} \leq M$.

Proof: Consider the Lyapunov function (11) as in Theorem 1. It follows from Theorem 1 that $V(\tilde{\omega}(t), \tilde{\mathcal{J}}(t)) \leq$ $V(\tilde{\omega}(0), \tilde{\mathcal{J}}(0))$ for all $t \geq 0$ and hence

$$
\begin{aligned}
& \tilde{\omega}^{\mathrm{T}}(t) J \tilde{\omega}(t)+\tilde{\mathcal{J}}^{\mathrm{T}}(t) Q^{-1} \tilde{\mathcal{J}}(t) \\
& \quad \leq \tilde{\omega}^{\mathrm{T}}(0) J \tilde{\omega}(0)+\tilde{\mathcal{J}}^{\mathrm{T}}(0) Q^{-1} \tilde{\mathcal{J}}(0) .
\end{aligned}
$$

Next, note that

$$
\begin{aligned}
\sigma_{\min }(J)\|\tilde{\omega}(t)\|_{2}^{2}+\frac{\|\tilde{\mathcal{J}}(t)\|_{2}^{2}}{\sigma_{\max }(Q)} \\
\quad \leq \tilde{\omega}^{\mathrm{T}}(t) J \tilde{\omega}(t)+\tilde{\mathcal{J}}^{\mathrm{T}}(t) Q^{-1} \tilde{\mathcal{J}}(t)
\end{aligned}
$$

and

$$
\begin{aligned}
\tilde{\omega}^{\mathrm{T}}(0) J \tilde{\omega}(0)+\tilde{\mathcal{J}}^{\mathrm{T}}(0) & Q^{-1} \tilde{\mathcal{J}}(0) \\
& \leq \sigma_{\max }(J)\|\tilde{\omega}(0)\|_{2}^{2}+\frac{\|\tilde{\mathcal{J}}(0)\|_{2}^{2}}{\sigma_{\min }(Q)} .
\end{aligned}
$$

Furthermore, since $\|\tilde{\omega}(0)\|_{2} \leq m_{1}$ and $\|\tilde{\mathcal{J}}(0)\|_{2} \leq m_{2}$

$$
\sigma_{\max }(J)\|\tilde{\omega}(0)\|_{2}^{2}+\frac{\|\tilde{\mathcal{J}}(0)\|_{2}^{2}}{\sigma_{\min }(Q)} \leq \sigma_{\max }(J) m_{1}^{2}+\frac{m_{2}^{2}}{\sigma_{\min }(Q)} .
$$

Thus, from (23), (24), (25), and (26), we obtain

$$
\sigma_{\min }(J)\|\tilde{\omega}(t)\|_{2}^{2}+\frac{\|\tilde{\mathcal{J}}(t)\|_{2}^{2}}{\sigma_{\max }(Q)} \leq \sigma_{\max }(J) m_{1}^{2}+\frac{m_{2}^{2}}{\sigma_{\min }(Q)}
$$

for all $t \geq 0$ and hence

$$
\begin{aligned}
\|\tilde{\omega}(t)\|_{2} & \leq \sqrt{\frac{\sigma_{\max }(J)}{\sigma_{\min }(J)} m_{1}^{2}+\frac{m_{2}^{2}}{\sigma_{\min }(Q) \sigma_{\min }(J)}} \\
& \leq \bar{m}_{1}
\end{aligned}
$$


and

$$
\begin{aligned}
\|\tilde{\mathcal{J}}(t)\|_{2} & \leq \sqrt{\sigma_{\max }(J) \sigma_{\max }(Q) m_{1}^{2}+\frac{\sigma_{\max }(Q)}{\sigma_{\min }(Q)} m_{2}^{2}} \\
& \leq \bar{m}_{2}
\end{aligned}
$$

where $\bar{m}_{1}$ and $\bar{m}_{2}$ are defined as in (21) and (22). Thus, $\|\tilde{\omega}(t)\|_{2} \leq \bar{m}_{1}$ and $\|\tilde{\mathcal{J}}(t)\|_{2} \leq \bar{m}_{2}$.

Now, the control torque $\tau$ in (8) can be written as

$$
\begin{aligned}
\tau(t)= & -K \tilde{\omega}(t)+(\tilde{\omega}(t)+\nu(t))^{\times} L(\tilde{\omega}(t)+\nu(t)) \\
& \times(\tilde{\mathcal{J}}(t)+\mathcal{J})+L(\dot{\nu}(t))(\tilde{\mathcal{J}}(t)+\mathcal{J}) .
\end{aligned}
$$

Thus, $\|\tilde{\tilde{\mathcal{J}}}(t)\|_{2} \leq \sigma_{\max }(K)\|\tilde{\omega}(t)\|_{2}+\|\tilde{\omega}(t)+\nu(t)\|_{2} \| L(\tilde{\omega}(t)+$ $\nu(t))(\tilde{\mathcal{J}}(t)+\mathcal{J})\left\|_{2}+\right\| L(\dot{\nu}(t))(\tilde{\mathcal{J}}(t)+\mathcal{J}) \|_{2}$. To obtain an upper bound on $\|\tau(t)\|_{2}$, we first find an upper bound on $\|L(a) v\|_{2}$, where $L(\cdot)$ is given by (4) and $a \in \mathbb{R}^{3}$ and $v \in \mathbb{R}^{6}$. Given $a=\left[\begin{array}{lll}a_{1} & a_{2} & a_{3}\end{array}\right]^{\mathrm{T}}$, express $L(a)$ in (4) as $L(a)=\left[L_{1}(a) L_{2}(a)\right]$, where

$$
L_{1}(a)=\left[\begin{array}{ccc}
a_{1} & 0 & 0 \\
0 & a_{2} & 0 \\
0 & 0 & a_{3}
\end{array}\right], \quad L_{2}(a)=\left[\begin{array}{ccc}
0 & a_{3} & a_{2} \\
a_{3} & 0 & a_{1} \\
a_{2} & a_{1} & 0
\end{array}\right] .
$$

Furthermore, express $v \in \mathbb{R}^{6}$ as $v=\left[v_{1}^{\mathrm{T}} v_{2}^{\mathrm{T}}\right]^{\mathrm{T}}$, where $v_{1}, v_{2} \in$ $\mathbb{R}^{3}$. Then $L(a) v=L_{1}(a) v_{1}+L_{2}(a) v_{2}$. Therefore, $\|L(a) v\|_{2} \leq$ $\left\|L_{1}(a)\right\|_{i}\left\|v_{1}\right\|_{2}+\left\|L_{2}(a)\right\|_{i}\left\|v_{2}\right\|_{2}$, where $\|\cdot\|_{i}$ is the induced 2-norm. Note that $\left\|L_{1}\right\|_{i}=\|a\|_{\infty} \leq\|a\|_{2}$.

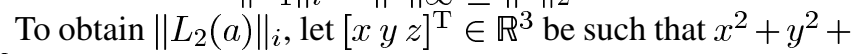
$z^{2}=1$. Then

$$
\left\|L_{2}(a)\right\|_{i}=\sup _{[x y z]^{\mathrm{T}} \in S^{2}}\left\|L_{2}(a)[x y z]^{\mathrm{T}}\right\|_{2} .
$$

Now

$$
\begin{aligned}
&\left\|L_{2}(a)[x y z]^{\mathrm{T}}\right\|_{2}^{2} \\
&= a_{3}^{2}\left(y^{2}+x^{2}\right)+a_{2}^{2}\left(z^{2}+x^{2}\right) \\
&+a_{1}^{2}\left(z^{2}+y^{2}\right) \\
&+2 a_{3} a_{2} y z+2 a_{3} a_{1} x z+2 a_{2} a_{1} x y, \\
&=\|a\|_{2}^{2}-\left(a_{3}^{2} z^{2}+a_{2}^{2} y^{2}+a_{1}^{2} x^{2}\right) \\
&+2 a_{3} a_{2} y z+2 a_{3} a_{1} x z+2 a_{2} a_{1} x y, \\
&=\|a\|_{2}^{2}+\left(a_{3}^{2} z^{2}+a_{2}^{2} y^{2}+a_{1}^{2} x^{2}\right) \\
&-\left(a_{3} z-a_{2} y\right)^{2}-\left(a_{3} z-a_{1} x\right)^{2}-\left(a_{2} y-a_{1} x\right)^{2}, \\
& \leq\|a\|_{2}^{2}+\left(a_{3}^{2} z^{2}+a_{2}^{2} y^{2}+a_{1}^{2} x^{2}\right) \leq 2\|a\|_{2}^{2} .
\end{aligned}
$$

Therefore, $\left\|L_{2}(a)\right\|_{i} \leq \sqrt{2}\|a\|_{2}$ and hence, $\|L(a) \cdot v\|_{2} \leq$ $\|a\|_{2}\left\|v_{1}\right\|_{2}+\sqrt{2}\|a\|_{2}\left\|v_{2}\right\|_{2} \leq \sqrt{2}\|a\|_{2}\left(\left\|v_{1}\right\|_{2}+\left\|v_{2}\right\|_{2}\right)$. Since, $\left\|v_{1}\right\|_{2}+\left\|v_{2}\right\|_{2} \leq \sqrt{3}\|v\|_{2},\|L(a) \cdot v\|_{2} \leq \sqrt{6}\|a\|_{2}\|v\|_{2}$.

Thus

$$
\begin{aligned}
\|\tau(t)\|_{2} \leq \sigma_{\max }(K)\|\tilde{\omega}(t)\|_{2}+\sqrt{6}\|\tilde{\omega}(t)+\nu(t)\|_{2}^{2} \\
\quad \times\|\tilde{\mathcal{J}}(t)+\mathcal{J}\|_{2}+\sqrt{6}\|\dot{\nu}(t)\|_{2}\|\tilde{\mathcal{J}}(t)+\mathcal{J}\|_{2} .
\end{aligned}
$$

Next, (27), (28), and the upper bounds on $\nu(t)$ and $\mathcal{J}$, yields $\|\tilde{\omega}(t)+\nu(t)\|_{2} \leq \bar{m}_{1}+\eta_{1}$ and $\|\tilde{\mathcal{J}}(t)+\mathcal{J}\|_{2} \leq \bar{m}_{2}+m_{\mathcal{J}}$. Therefore, for all $t \geq 0$

$$
\begin{aligned}
\|\tau(t)\|_{2} \leq \sigma_{1}(K) \bar{m}_{1} & +\sqrt{6}\left(\bar{m}_{1}+\eta_{1}\right)^{2} \\
& \times\left(\bar{m}_{2}+m_{\mathcal{J}}\right)+\sqrt{6}\left(\bar{m}_{2}+m_{\mathcal{J}}\right) \eta_{2}=M
\end{aligned}
$$

and the result follows.

If the upper bounds $\eta_{1}, \eta_{2}, m_{1}, m_{2}$, and $m_{\mathcal{J}}$ are known, then $M$ in Theorem 3 can be computed. Thus, Theorem 3 implies that as long as the actuators can provide a torque of magnitude $M$, the controller given by (7) and (8) can track any reference command signal that satisfies $\|\nu(t)\|_{2} \leq \eta_{1}$ and $\|\dot{\nu}(t)\|_{2} \leq \eta_{2}$, for all $t \geq 0$. Thus, $M$ provides a minimum saturation level for the applied torques.

Remark 1: The bound $M$ computed in Theorem 3 is conservative since the maximum required torque level can be much lower than $M$. Theorem 3, however, demonstrates that the controller given by (7) and (8) can function even under saturation effects, and $M$ represents an upper bound for the required saturation level.

\section{Inertia Identification Using Periodic REFERENCE SIGNALS}

The following corollaries of Lemma 1 consider the special case in which $\nu$ is constant. The proof is similar to results presented in [19]. Define

$$
G_{0}(\nu) \triangleq\left\{\chi \in \mathbb{R}^{6}: \nu^{\times} L(\nu) \chi=0\right\} .
$$

Corollary 1: Assume that $\nu$ is constant. Under the control law (7) and (8), $\tilde{\mathcal{J}}(t) \rightarrow G_{0}(\nu)$ as $t \rightarrow \infty$.

In Corollary $1, \nu$ represents a constant spin about a body-fixed axis. In the case that this body-fixed axis is the principal axis, it is equivalent to $\nu^{\times} J \nu=0$. For such a case, we expect that $\tau \rightarrow 0$ as $t \rightarrow \infty$. The following result shows that the control law (7) and (8) indeed has this property.

Corollary 2: Assume that $\nu$ is constant and satisfies $\nu^{\times} J \nu=$ 0 . Then, under the control law (7) and (8), it follows that $\tau \rightarrow 0$ as $t \rightarrow \infty$.

We now apply Corollary 1 to identify the off-diagonal terms $J_{12}, J_{23}$, and $J_{31}$.

Proposition 1: Let $\nu$ be constant. If $\nu=\left[\nu_{1} 00\right]^{\mathrm{T}}$, where $\nu_{1} \neq 0$, then, under the control law (7) and (8), $\hat{J}_{13} \rightarrow J_{13}$ and $\hat{J}_{12} \rightarrow J_{12}$ as $t \rightarrow \infty$. Furthermore, if $\nu=\left[0 \nu_{2} 0\right]^{\mathrm{T}}$, where $\nu_{2} \neq 0$, then, under the control law (7) and (8), $\hat{J}_{23} \rightarrow J_{23}$ and $\hat{J}_{12} \rightarrow J_{12}$ as $t \rightarrow \infty$.

Theorem 2 provides sufficient conditions on the reference signal $\nu$ that guarantee asymptotic identification of the inertia matrix. In particular, the reference signal need not be periodic. However, in the case of a periodic reference signal, we obtain a stronger result.

Proposition 2: Let $\nu$ be periodic with period $T_{0}$ and suppose there exist $0 \leq \hat{t}_{1} \leq \hat{t}_{2} \leq, \ldots, \leq \hat{t}_{6}$ such that rank $\left(\mathcal{W}\left(\hat{t}_{1}, \ldots, \hat{t}_{6}\right)\right)=6$. Then, under the control law (7) and (8), $\hat{\mathcal{J}}(t) \rightarrow \mathcal{J}$ as $t \rightarrow \infty$. 
Proof: $\operatorname{Since} \operatorname{rank}\left(\mathcal{W}\left(\hat{t}_{1}, \ldots, \hat{t}_{6}\right)\right)=6$, there exists $\gamma>0$ such that $\sigma_{\min }\left(\mathcal{W}\left(\hat{t}_{1}, \ldots, \hat{t}_{6}\right)\right)>\gamma$. Choose $\delta=2 T_{0}$. Next, we show that for all $T \geq 0$, there exist $t_{1}, \ldots, t_{6} \in[T, T+\delta]$ such that $\sigma_{\min }\left(\mathcal{W}\left(t_{1}, \ldots, t_{6}\right)\right)>\gamma$.

Define $\operatorname{rem}(x, y)$ as the remainder obtained when $x$ is divided by $y$, where $x, y \in \mathbb{R}$. Then, $T \geq 0$ can be expressed as $T=$ $m T_{0}+\operatorname{rem}\left(T, T_{0}\right)$, for some non-negative integer $m$. Choose $t_{i} \triangleq(m+1) T_{0}+\operatorname{rem}\left(\hat{t}_{i}, T_{0}\right)$, for all $i \in\{1, \ldots, 6\}$. Clearly, $\hat{t}_{i} \geq T$ and

$$
\begin{aligned}
\left|t_{i}-T\right| & \leq\left|(m+1) T_{0}-T\right|+\operatorname{rem}\left(T, T_{0}\right), \\
& =\left|T_{0}-\operatorname{rem}\left(T, T_{0}\right)\right|+\operatorname{rem}\left(T, T_{0}\right), \\
& \leq T_{0}+\operatorname{rem}\left(T, T_{0}\right) \leq 2 T_{0}=\delta .
\end{aligned}
$$

Thus, $t_{i} \in[T, T+\delta]$, for all $i \in\{1, \ldots, 6\}$. Finally, since $\nu$ has a time period $T_{0}$, it follows that $\nu\left(t_{i}\right)=\nu\left(\operatorname{rem}\left(\hat{t}_{i}, T_{0}\right)\right)=$ $\nu\left(\hat{t}_{i}\right)$. Thus, $\mathcal{W}\left(t_{1}, \ldots, t_{6}\right)=\mathcal{W}\left(\hat{t}_{1}, \ldots, \hat{t}_{6}\right)$ and hence, $\sigma_{\min }\left(\mathcal{W}\left(t_{1}, \ldots, t_{6}\right)\right)>\gamma$. Thus, for all $T \geq 0$, there exist $t_{1}, \ldots, t_{6} \in[T, T+\delta]$ such that $\sigma_{\min }\left(\mathcal{W}\left(t_{1}, \ldots, t_{6}\right)\right)>\gamma$. The result now follows from Theorem 2 .

\section{Simulation Results}

To illustrate Theorem 1, we demonstrate the results in Propositions 1 and 2, which demonstrate the convergence of the inertia parameters to their true values, as well as, convergence of the angular velocity to the reference angular velocity. The moment of inertia chosen for the model is given as

$$
J=\left[\begin{array}{ccc}
25 & 1.2 & 0.9 \\
1.2 & 17 & 1.4 \\
0.9 & 1.4 & 15
\end{array}\right] \mathrm{kg} / \mathrm{m}^{2} .
$$

For simulations demonstrating Proposition 1, the gains for the controller are chosen to be $K=20 I_{3}$ and $Q=300 I_{6}$, and the initial condition for $\omega$ is chosen such that $\tilde{\omega}(0)=[333]^{\tau}$ corresponding to the two reference angular velocity signals given in Proposition 1, where each of $\nu_{1}$ and $\nu_{2}$ are chosen to be 0.5 . The gains $K$ and $Q$ for the controller, were chosen by trial-and-error to obtain reasonable convergence rates. Also

$$
\hat{J}(0)=\left[\begin{array}{ccc}
25 & 0.6 & 0.5 \\
0.6 & 12 & 2 \\
0.5 & 2 & 10
\end{array}\right] \mathrm{kg} / \mathrm{m}^{2} .
$$

The first reference angular velocity to be tracked is given by $\nu=\left[\begin{array}{lll}0.5 & 0 & 0\end{array}\right]^{\mathrm{T}} \mathrm{rad} / \mathrm{s}$. As guaranteed by Proposition 1, the estimates $\hat{J}_{13}$ and $\hat{J}_{12}$, converge to the true values $J_{13}$ and $J_{12}$, respectively, as shown in Fig. 1. However, $\hat{J}_{23}$ is not guaranteed to converge to $J_{23}$, and as shown in Fig. 1, does not. Also, Theorem 1 implies that the angular velocity converges to the reference angular velocity $\nu$. The corresponding numerical results are shown in Fig. 2.

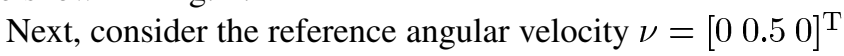
$\mathrm{rad} / \mathrm{sec}$. In this case, Proposition 1 implies that the off-diagonal terms $J_{23}$ and $J_{12}$ converge to their true values. Again, note that the estimate for the off-diagonal entry $J_{13}$ does not converge to
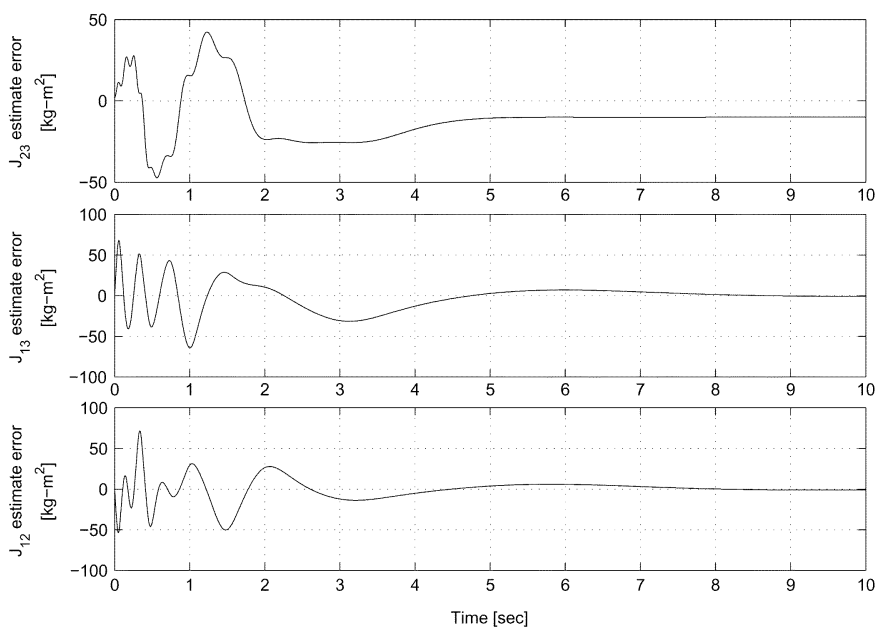

Fig. 1. Identification of off-diagonal terms in the inertia matrix using $\nu=$ $\left[\begin{array}{lll}0.5 & 0 & 0\end{array}\right]^{\mathrm{T}}$.
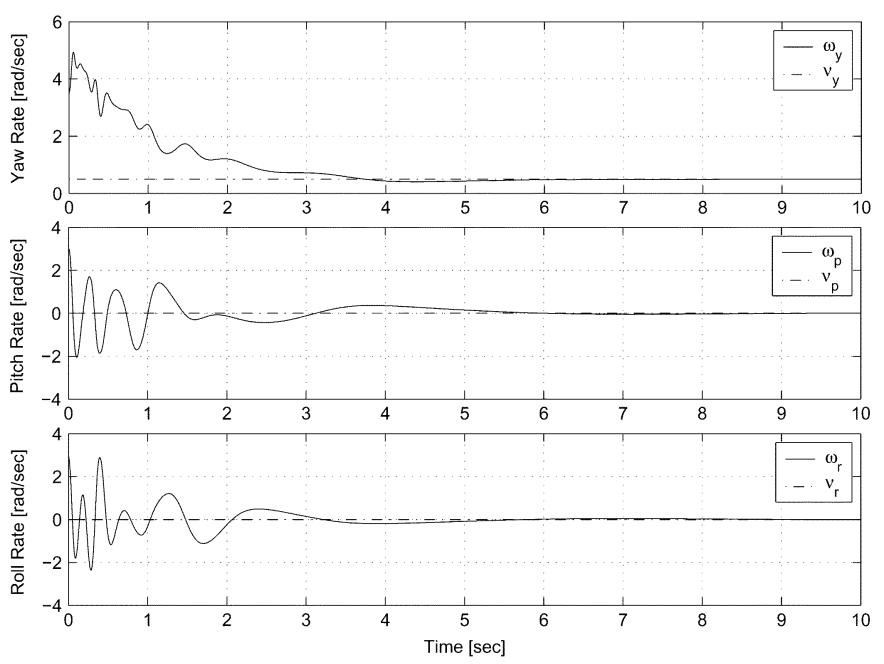

Fig. 2. Angular velocity tracking for $\nu=\left[\begin{array}{lll}0.5 & 0 & 0\end{array}\right]^{\mathrm{T}}$.

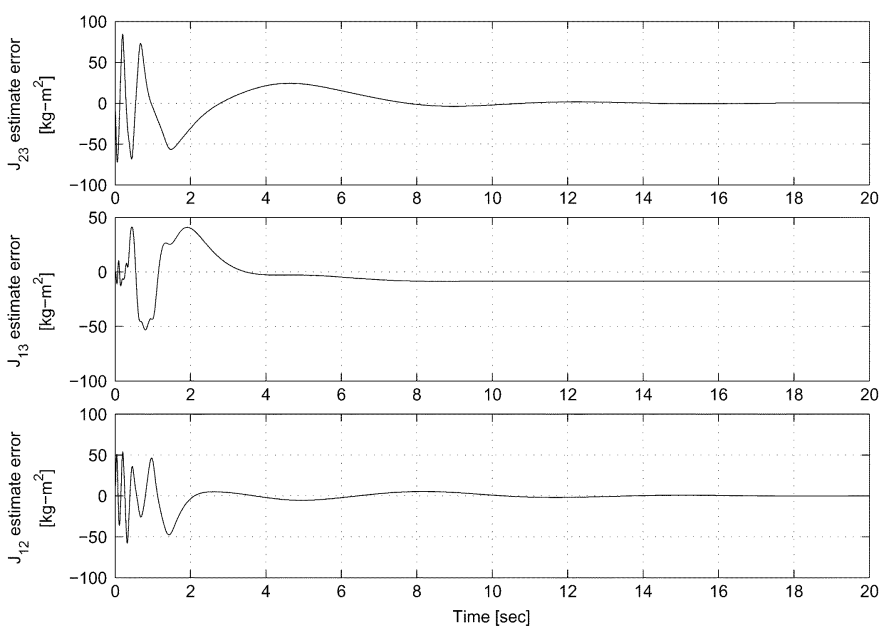

Fig. 3. Identification of off-diagonal terms in the inertia matrix using $\nu=$ $\left[\begin{array}{lll}0 & 0.5 & 0\end{array}\right]^{\mathrm{T}}$.

its true value, and the angular velocity converges to the reference $\nu=\left[\begin{array}{lll}0 & 0.5 & 0\end{array}\right]^{\mathrm{T}} \mathrm{rad} / \mathrm{s}$ as seen in Figs. 3 and 4. Hence, the off- 

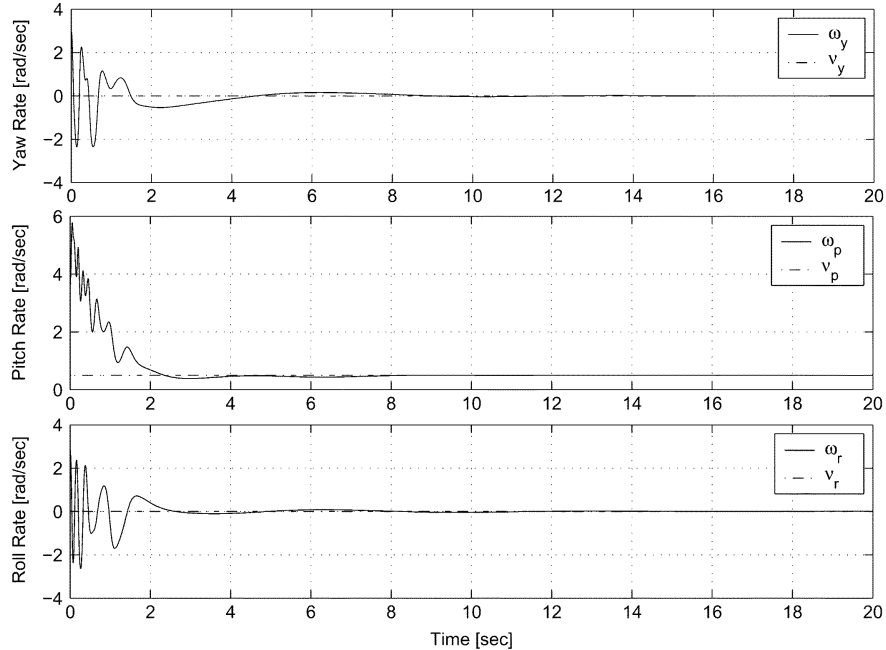

Fig. 4. Angular velocity tracking for $\nu=\left[\begin{array}{lll}0 & 0.5 & 0\end{array}\right]^{\mathrm{T}}$.
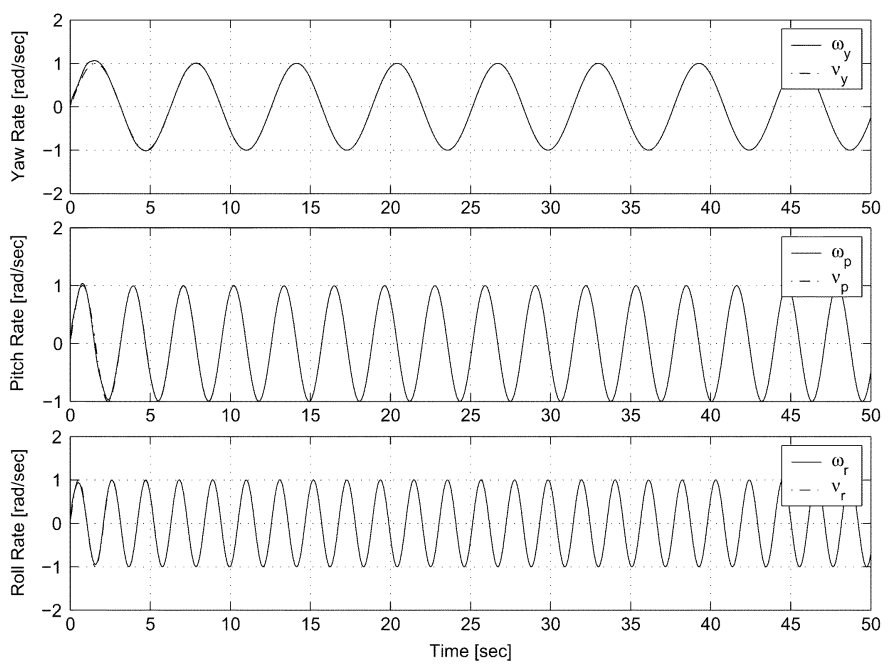

Fig. 5. Angular velocity tracking for $\nu(t)=[\sin t \sin 2 t \sin 3 t]^{\mathrm{T}}$.

diagonal terms $J_{12}, J_{13}$, and $J_{23}$ can be identified by performing two constant tracking maneuvers.

We now consider periodic maneuvers for identifying the entire inertia matrix. To satisfy the conditions of Proposition 2, consider the periodic reference angular velocity $\nu(t)=[\sin t \sin 2 t \sin 3 t]^{\mathrm{T}} \mathrm{rad} / \mathrm{s}$. Then, with $t_{1}=0$ and $t_{2}=\pi / 2$, we obtain

$$
\mathcal{W}(0, \pi / 2) \triangleq\left[\begin{array}{c}
W(0) \\
W(\pi / 2)
\end{array}\right]=\left[\begin{array}{cccccc}
1 & 0 & 0 & 0 & 3 & 2 \\
0 & 2 & 0 & 3 & 0 & 1 \\
0 & 0 & 3 & 2 & 1 & 0 \\
0 & 0 & 0 & -1 & 0 & -1 \\
-1 & -2 & 1 & 0 & 0 & 0 \\
0 & 0 & 0 & -3 & 0 & 1
\end{array}\right] .
$$

The maximum and minimum singular values of $\mathcal{W}(0, \pi / 2)$ are 5.2406 and 0.3512 , respectively. Therefore, rank $\mathcal{W}(0, \pi / 2)=$ 6 . Hence, Theorem 1 and Proposition 2 guarantee that, under the control law (7) and (8), $\tilde{\omega}(t) \rightarrow 0$ and $\hat{\mathcal{J}}(t) \rightarrow \mathcal{J}$. We choose $K=150 I_{3}, Q=100 I_{6}$, and $\omega(0)=0$ for simulations, and
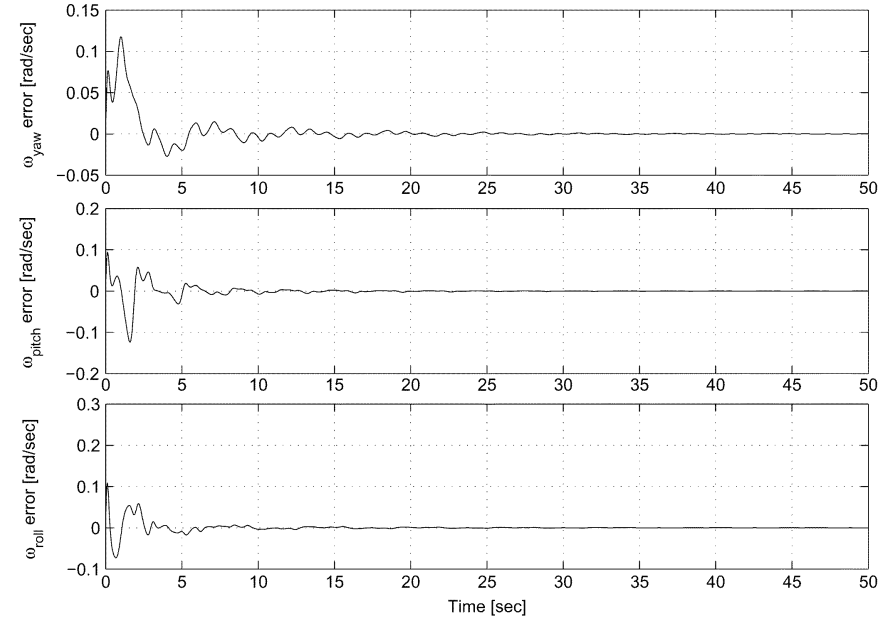

Fig. 6. Error in angular velocity tracking for $\nu(t)=[\sin t \sin 2 t \sin 3 t]^{\mathrm{T}}$.
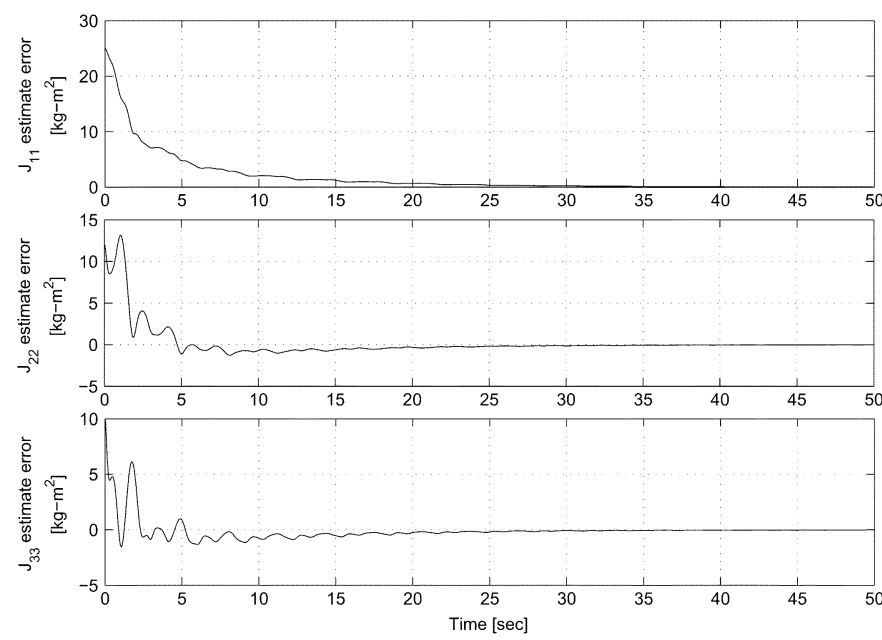

Fig. 7. Error in identification of diagonal terms in the inertia matrix.

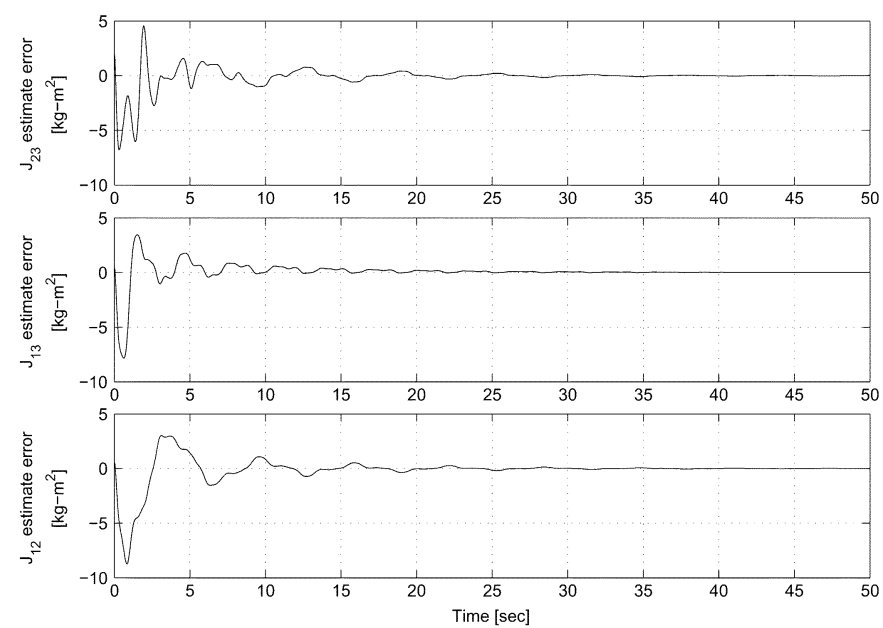

Fig. 8. Error in identification of off-diagonal terms in the inertia matrix.

$\hat{\mathcal{J}}(0)$ is chosen as before. Figs. 5 and 6 show that $\tilde{\omega}(t) \rightarrow 0$ as $t \rightarrow \infty$, while Figs. 7 and 8 indicate that $\hat{\mathcal{J}}(t) \rightarrow \mathcal{J}(t)$ in accordance with Proposition 2. The control effort is shown in Fig. 9. 


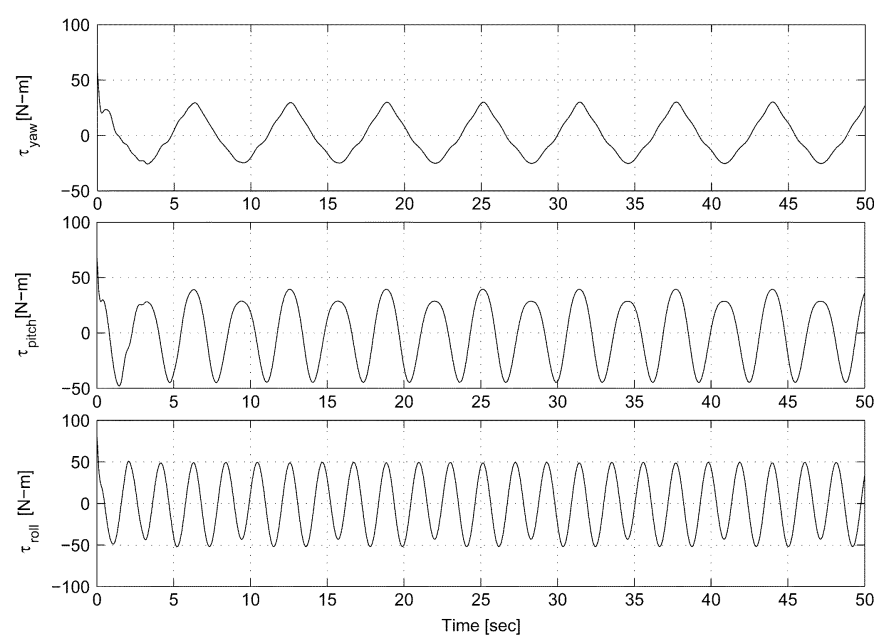

Fig. 9. Control torques applied to $\operatorname{track} \nu(t)=[\sin t \sin 2 t \sin 3 t]^{\mathrm{T}}$.

\section{EXPERIMENTAL SETUP}

In this section, we discuss implementation issues, as well as, modeling of the actuator moments in the experimental setup. Our experimental implementation uses the Triaxial Attitude Control Testbed (TACT) in the Attitude Dynamics and Control Laboratory of the University of Michigan, Ann Arbor. This testbed is described in [1], [21], and [22].

The experimental testbed is based on a spherical air bearing manufactured by Space Electronics, Inc., Berlin, CT. An 11-in diameter aluminum sphere floats on a thin film of air that exits from holes located in the surface of the cup. Air at $70 \mathrm{psi}$ is supplied to the cup by means of a hose that passes through the center of the vertical support. The spherical air bearing allows unrestricted motion in yaw (rotation about the vertical axis) and roll (rotation about the longitudinal shaft axis). The plates and shafts are designed to allow $\pm 45^{\circ}$ pitch (rotation about a horizontal axis) at all roll and yaw angles.

Once the main components are mounted, additional masses can be added to modify the mass distribution. This mass distribution balances pitch motion. However, when the center of mass is not located at the rotational center, the body possesses pendulum dynamics [23]. In the experimental results reported herein, the center of mass of the supported body is assumed to be located at the pivot point. This balancing implies that there are no gravitational moments on the body so that the body is modeled by the equations of motion given in (1).

Fig. 10 shows a picture of the TACT and Fig. 11 shows a schematic of the relative positions of the thrusters. The TACT has four thrusters, each of which has nonlinear input-output (I/O) characteristics. In [1], an adaptive feedback-linearizationbased controller was designed to handle this nonlinearity. In the present paper, for simplicity, we identify the nonlinearity and invert it in the controller. A maximum torque of about $20 \mathrm{~N} \cdot \mathrm{m}$ can be generated using these thrusters.

Figs. 12 and 13 represent the forces due to the thrusters and the corresponding torques about the pivot point. Let $V_{f_{i}}$ denote the voltage applied to the $i$ th thruster, and let $F_{i}$ and $\tau_{i}$ denote the

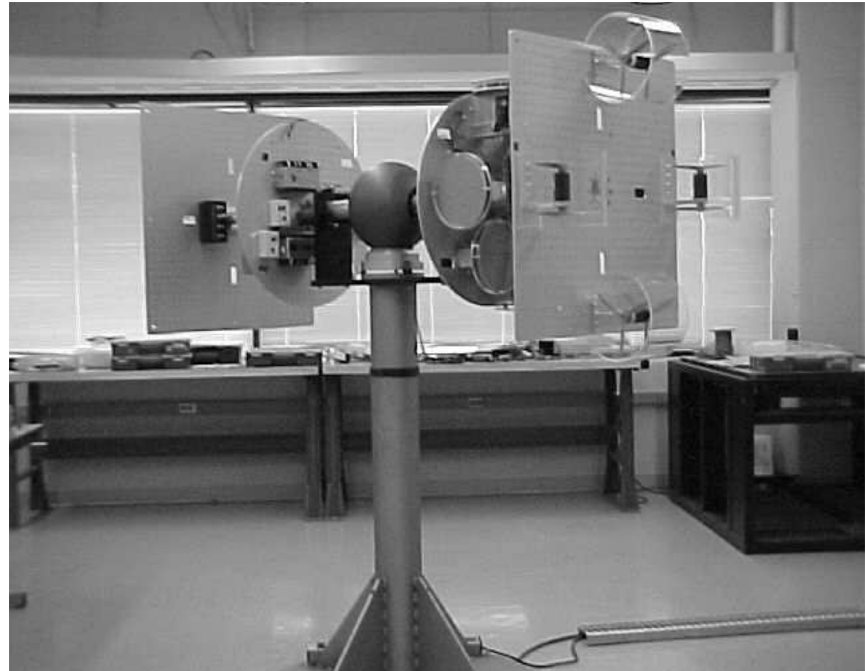

Fig. 10. TACT. This testbed, which is based on a spherical air bearing, allows low friction, 3 -D motion with unrestricted roll and yaw and $\pm 45^{\circ}$ pitch.

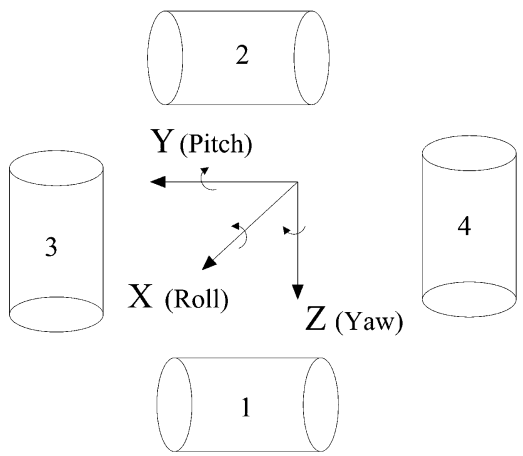

Fig. 11. Orientation of thrusters on the TACT.

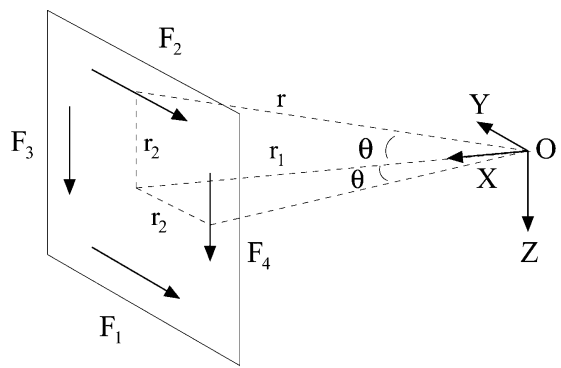

Fig. 12. Forces due to thrusters on the TACT.

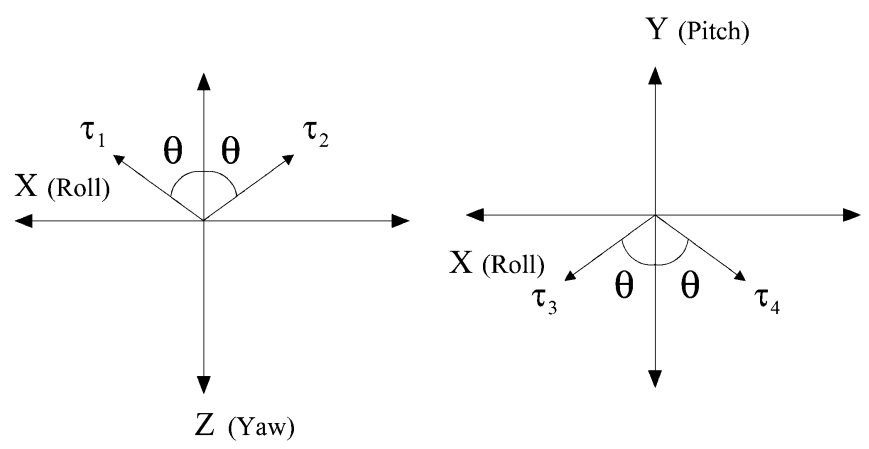

Fig. 13. Torque applied due to thrusters on the TACT. 


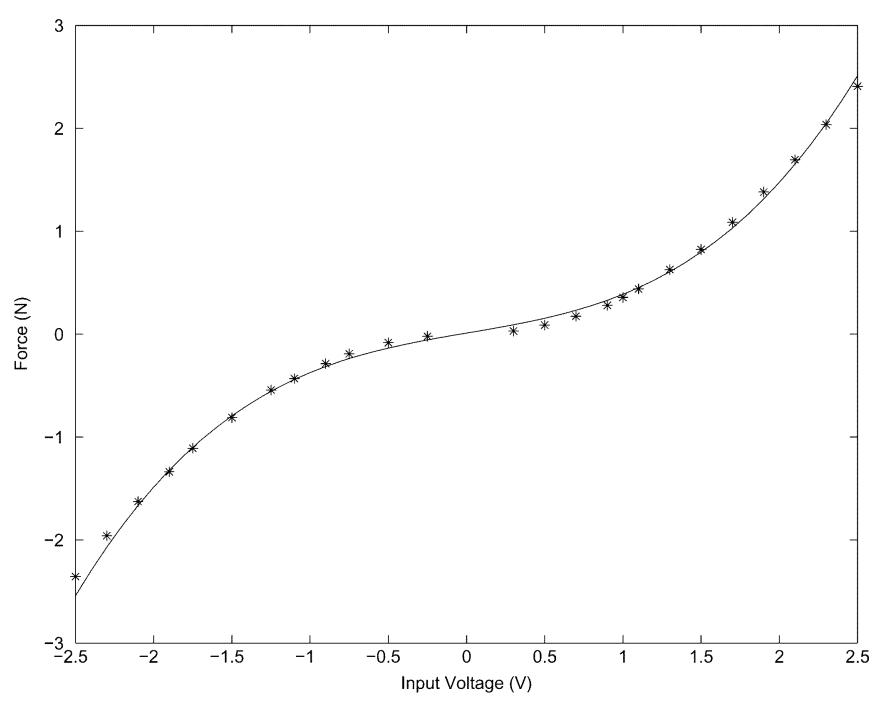

Fig. 14. Data for force versus input voltage for thrusters 1 and $2\left(\mathcal{N}_{y}\right)$.

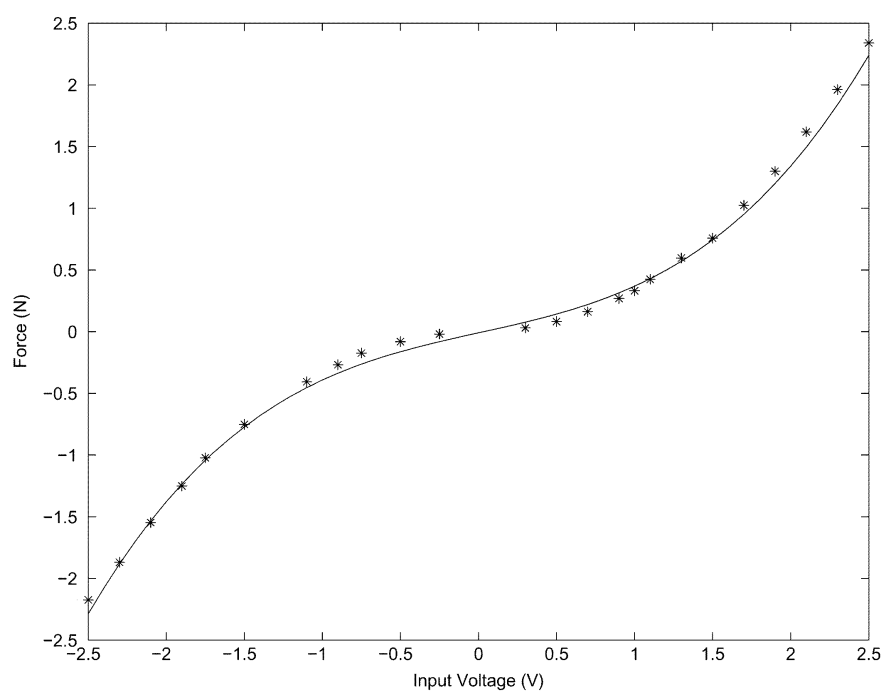

Fig. 15. Data for force versus input voltage for thrusters 3 and $4\left(\mathcal{N}_{p}\right)$.

force and torque due to the $i$ th thruster, where $i \in\{1,2,3,4\}$. It can be seen that the net torque due to the thrusters is given by

$$
\begin{aligned}
\tau_{\text {yaw }} & =-\left(\tau_{1}+\tau_{2}\right) \cos \theta \\
\tau_{\text {pitch }} & =-\left(\tau_{3}+\tau_{4}\right) \cos \theta \\
\tau_{\text {roll }} & =\left[\left(\tau_{1}-\tau_{2}\right)+\left(\tau_{3}-\tau_{4}\right)\right] \sin \theta
\end{aligned}
$$

where $\tau_{i}=r F_{i}, r$ is the length and $\theta$ is the angle as shown in Fig. 12.

Experiments were performed on the TACT to determine the force generated by the thruster as a function of voltage. These experiments involve balancing the TACT by masses for a given thruster voltage, and then computing the force of the thruster from the torque required to balance the masses. Figs. 14 and 15 represent the one-to-one mappings obtained between the input voltage applied to a thruster and the resulting thrust force, for thruster pairs $(1,2)$ and $(3,4)$, respectively. The nonlinear maps in Figs. 14 and 15 were obtained by fitting a third order polynomial using least squares. The approximate expressions, $\mathcal{N}_{y}(x)$ and $\mathcal{N}_{p}(x)$ for each of the thruster in the thruster pairs $(1,2)$ and $(3,4)$, respectively, are given by

$$
\begin{aligned}
& \mathcal{N}_{y}(x)=0.12 x^{3}-0.004 x^{2}+0.26 x+0.01 \\
& \mathcal{N}_{p}(x)=0.1 x^{3}-0.0023 x^{2}+0.28 x-0.01
\end{aligned}
$$

where $x$ is in volts and $\mathcal{N}_{y}(x)$ and $\mathcal{N}_{p}(x)$ are in newtons. In other words, $F_{1}(x)=F_{2}(x)=\mathcal{N}_{y}(x)$ and $F_{3}(x)=F_{4}(x)=$ $\mathcal{N}_{p}(x)$.

Now $\tau_{i}=r F_{i}, i \in\{1,2,3,4\}$, where $r$ is the length as shown in Fig. 12. Substituting this in (30)-(32) and expressing $F_{i}, i \in$ $\{1,2,3,4\}$ in terms of $\mathcal{N}_{y}(x)$ and $\mathcal{N}_{p}(x)$ yields expressions for torques along the body axes given by

$$
\begin{aligned}
\tau_{\text {yaw }}= & -r_{1}\left[\mathcal{N}_{y}\left(V_{f_{1}}\right)+\mathcal{N}_{y}\left(V_{f_{2}}\right)\right] \\
\tau_{\text {pitch }}= & -r_{1}\left[\mathcal{N}_{p}\left(V_{f_{3}}\right)+\mathcal{N}_{p}\left(V_{f_{4}}\right)\right] \\
\tau_{\text {roll }}= & r_{2}\left(\left[\mathcal{N}_{y}\left(V_{f_{1}}\right)-\mathcal{N}_{y}\left(V_{f_{2}}\right)\right]\right. \\
& \left.+\left[\mathcal{N}_{p}\left(V_{f_{3}}\right)-\mathcal{N}_{p}\left(V_{f_{4}}\right)\right]\right)
\end{aligned}
$$

where the lengths $r_{1}=0.945 \mathrm{~m}$ and $r_{2}=0.33 \mathrm{~m}$.

Note that $\tau_{\text {yaw }}, \tau_{\text {pitch }}$, and $\tau_{\text {roll }}$ are computed by the adaptive algorithm. Furthermore, (35)-(37) are linear equations with $\mathcal{N}_{y}\left(V_{f_{1}}\right), \mathcal{N}_{y}\left(V_{f_{2}}\right), \mathcal{N}_{p}\left(V_{f_{3}}\right)$, and $\mathcal{N}_{p}\left(V_{f_{4}}\right)$ as unknowns, and that $\mathcal{N}_{y}(\cdot)$ and $\mathcal{N}_{p}(\cdot)$ are known invertible functions. Since there are four unknowns and three equations, there are an infinite number of solutions for the corresponding thruster voltages. These solutions represent alternative actuation schemes that can be implemented using four thrusters to generate three independent torques.

Out of the possible solutions, we select the scheme wherein the load for the roll moment is shared symmetrically between the thruster pairs $(1,2)$ and $(3,4)$. This choice results in the constraint equation

$$
\mathcal{N}_{y}\left(V_{f_{1}}\right)-\mathcal{N}_{y}\left(V_{f_{2}}\right)=\mathcal{N}_{p}\left(V_{f_{3}}\right)-\mathcal{N}_{p}\left(V_{f_{4}}\right) .
$$

Then, solving for the four unknowns in (35)-(37), using (38), and inverting the nonlinear map yields the thruster voltages

$$
\begin{aligned}
& V_{f_{1}}=\mathcal{N}_{y}^{-1}\left(-\frac{\tau_{\text {yaw }}}{2 r_{1}}+\frac{\tau_{\text {roll }}}{4 r_{2}}\right) \\
& V_{f_{2}}=\mathcal{N}_{y}^{-1}\left(-\frac{\tau_{\text {yaw }}}{2 r_{1}}-\frac{\tau_{\text {roll }}}{4 r_{2}}\right) \\
& V_{f_{3}}=\mathcal{N}_{p}^{-1}\left(-\frac{\tau_{\text {pitch }}}{2 r_{1}}+\frac{\tau_{\text {roll }}}{4 r_{2}}\right) \\
& V_{f_{4}}=\mathcal{N}_{p}^{-1}\left(-\frac{\tau_{\text {pitch }}}{2 r_{1}}-\frac{\tau_{\text {roll }}}{4 r_{2}}\right) .
\end{aligned}
$$

\section{EXPERIMENTAL RESULTS}

In this section, we implement the controller (7) and (8) on the TACT testbed. To demonstrate the effectiveness of the control algorithm, we perform two sets of experiments. In the first set, 
we track a reference angular velocity, starting from an initial angular velocity provided by giving the TACT an initial push. The aim of this set of experiments is to demonstrate the angular velocity tracking capability of the designed controller.

In the next set of experiments, the aim is to demonstrate the parameter identification capability of the control algorithm. These experiments are guided by the conditions given in propositions 1 and 2. Whereas Proposition 1 requires a constant reference angular velocity $\nu$ about one of the body axes, Proposition 2 requires a periodic angular velocity $\nu$ along all three body axes. Unfortunately, this requirement from Proposition 2 is stringent for two reasons. First, it is difficult to balance the TACT so that its center of gravity lies exactly at the pivot point. Second, the support of the TACT restricts the free motion in pitch, and hence, limits the class of angular velocity signals that can be given to the controller to track.

Thus, for the reasons mentioned above, it is difficult to demonstrate Proposition 2. However, we demonstrate Proposition 1 in the second set of experiments, where a constant angular velocity reference is given and the off-diagonal inertia estimates are computed.

Again, due to the presence of an onboard computer, sensors and the associated electronics and the electrical wiring, the moment of inertia of the TACT is unknown. This limits our ability to simulate a configuration that is close to the TACT.

\section{A. Angular Velocity Tracking}

As mentioned above, we push the TACT to provide an initial angular velocity, and command a constant angular velocity reference to the controller. The chosen reference angular velocity is $\nu=[300-3 \sin (0.25 t)]^{\mathrm{T} \%} / \mathrm{s}$, and the controller gains are chosen to be

$$
K=\left[\begin{array}{ccc}
30 & 0 & 0 \\
0 & 75 & 0 \\
0 & 0 & 10
\end{array}\right] \quad Q=100 \times \operatorname{diag}(1,1,1,7,1,1)
$$

Furthermore, $\hat{J}(0)=\operatorname{diag}(50,50,5)$.

We now compute the bounds $\bar{m}_{1}, \bar{m}_{2}$, and $M$ given by Theorem 3. Corresponding to the reference signals we choose $\eta_{1} \approx 30^{\circ} / \mathrm{s}=0.52 \mathrm{rad} / \mathrm{s}$ and $\eta_{2} \approx 0.75^{\circ} / \mathrm{s}^{2}=0.0131$ $\mathrm{rad} / \mathrm{s}^{2}$. Also, $m_{\mathcal{J}}=75 \mathrm{~kg} \cdot \mathrm{m}^{2}$. Since we start from rest, $m_{1}=0.52 \mathrm{rad} / \mathrm{s}$, and assuming that our estimates for the principal moment of inertia are close to about $5 \mathrm{~kg} \cdot \mathrm{m}^{2}$, we choose $m_{2}=10 \mathrm{~kg} \cdot \mathrm{m}^{2}$.

Now, choose $\sigma_{J}^{\mathrm{sup}}=50 \mathrm{~kg} \cdot \mathrm{m}^{2}, \sigma_{J}^{\mathrm{inf}}=5 \mathrm{~kg} \cdot \mathrm{m}^{2}$, and note that $\sigma_{\min }(Q)=100, \sigma_{\max }(Q)=700$, and $\sigma_{\max }(K)=75$. Then computing from (21), (22), and (20), we obtain $\bar{m}_{1}=1.7151$ $\mathrm{rad} / \mathrm{s} \approx 100^{\circ} / \mathrm{s}, \bar{m}_{2}=101.5 \mathrm{~kg} \cdot \mathrm{m}^{2}$, and $M \approx 1990 \mathrm{~N} \cdot \mathrm{m}$. Note that this is a conservative estimate, and as we see subsequently, our controller performs satisfactorily with actuators having a much lower torque capacity of about $20 \mathrm{~N} \cdot \mathrm{m}$.

The angular velocity tracking error, plotted in Fig. 16, shows that the yaw angular velocity error converges to zero quickly, whereas the error in pitch converges to zero relatively slowly. This suggests an increase in the feedback gain in $K$ corresponding to pitch oscillations. However, attempts at this caused large transients, in which the amplitude of the pitch oscillation
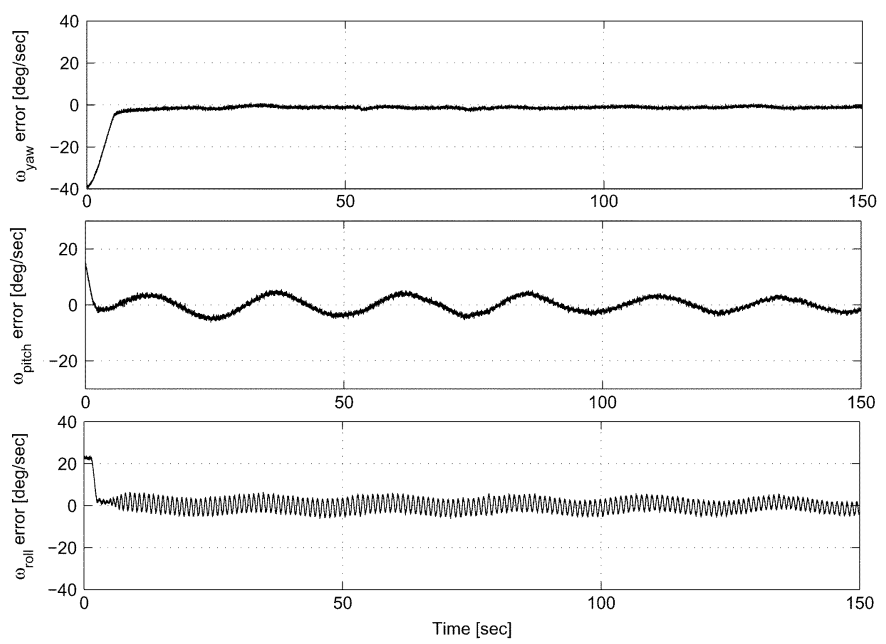

Fig. 16. Error in angular velocity for $\nu(t)=[300-3 \sin (0.25 t)] \mathrm{T}$. The roll oscillation of amplitude $0.5^{\circ}$ is due to imperfect balance of the TACT.
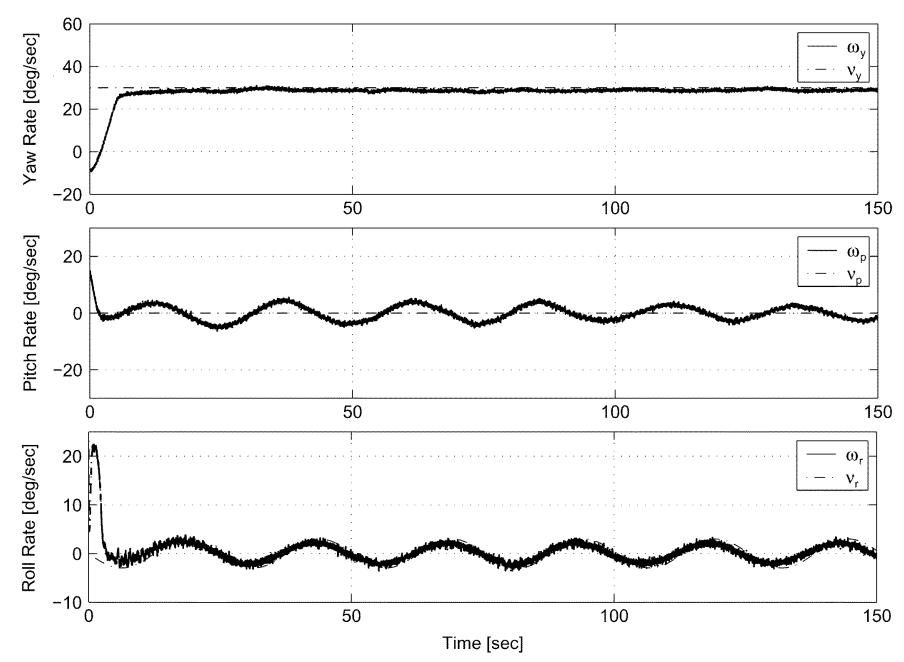

Fig. 17. Angular velocity tracking for $\nu(t)=[300-3 \sin (0.25 t)]^{\mathrm{T}}$.

was close to $45^{\circ}$ pitch constraint. Nevertheless, it is clear that the amplitude of the oscillations in the pitch decrease with time after $75 \mathrm{~s}$.

In roll response, the angular velocity error is modulated by a high frequency component. A power spectrum analysis of the signal reveals a harmonic component with a frequency of about $6.5 \mathrm{rad} / \mathrm{s}$ and amplitude $0.5^{\circ}$. The only frequency component of $\nu(t)$ is $0.25 \mathrm{rad} / \mathrm{s}$, and hence, the ratio of the noise frequency to the reference signal is approximately 25 . This oscillation was not discernible to the human eye. The roll oscillations are believed to be due to imperfect balancing of the TACT, leading to pendulum-type behavior due to gravitational moments [23].

Next, the roll data are filtered and the angular velocity error is shown in Fig. 18. The angular velocities are shown in Fig. 17. From this plot, it is clear that the angular velocity converges to the reference angular velocity, even for large initial errors, thus, demonstrating the global tracking ability of the controller.

\section{B. Inertia Parameter Identification}

In this section, we demonstrate the parameter identification ability of the controller (7) and (8). Specifically, by means of 

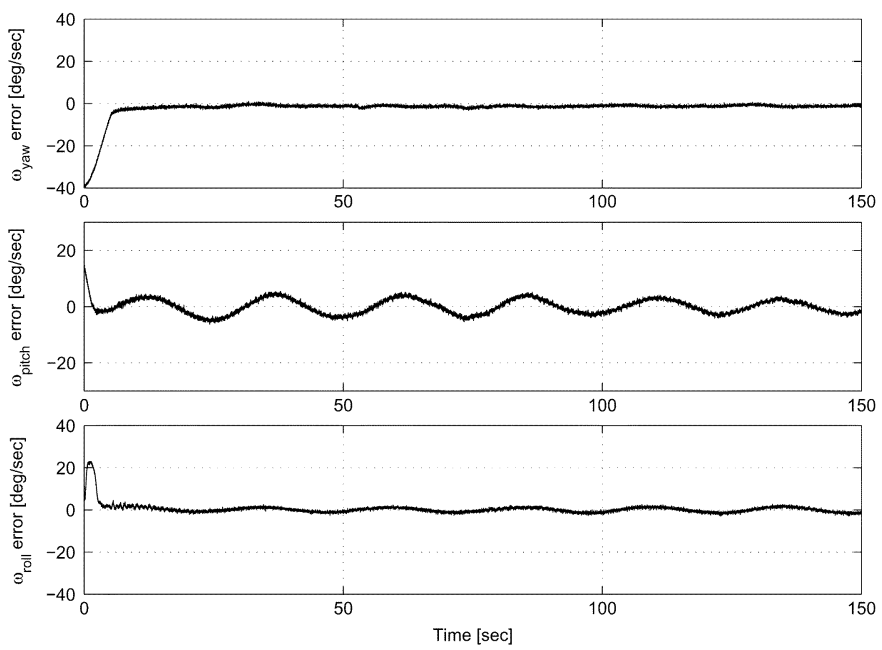

Fig. 18. Error in angular velocity for $\nu(t)=[300-3 \sin (0.25 t)]^{\mathrm{T}}$
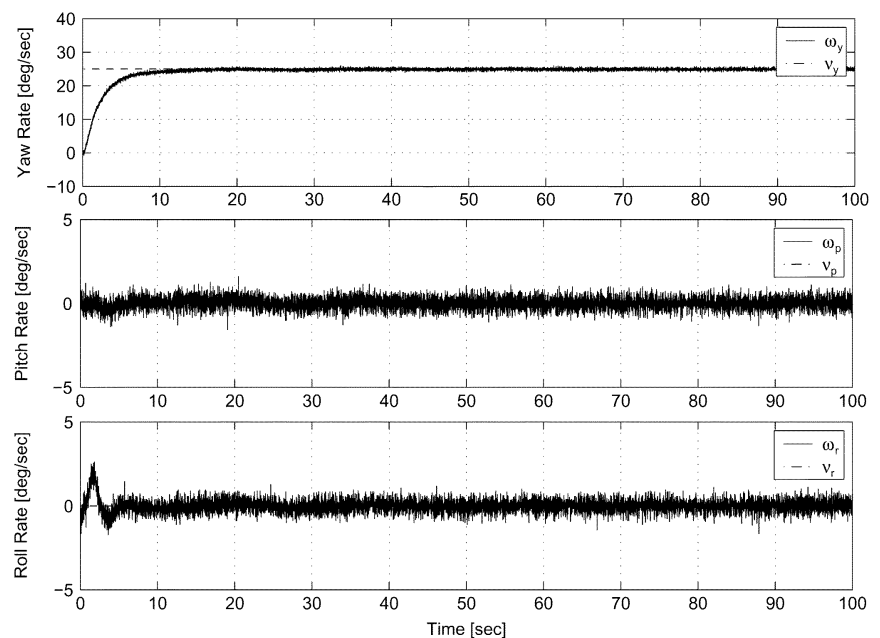

Fig. 19. Angular velocity tracking for $\nu_{1}=\left[\begin{array}{lll}25 & 0 & 0\end{array}\right]^{\mathrm{T}}$.
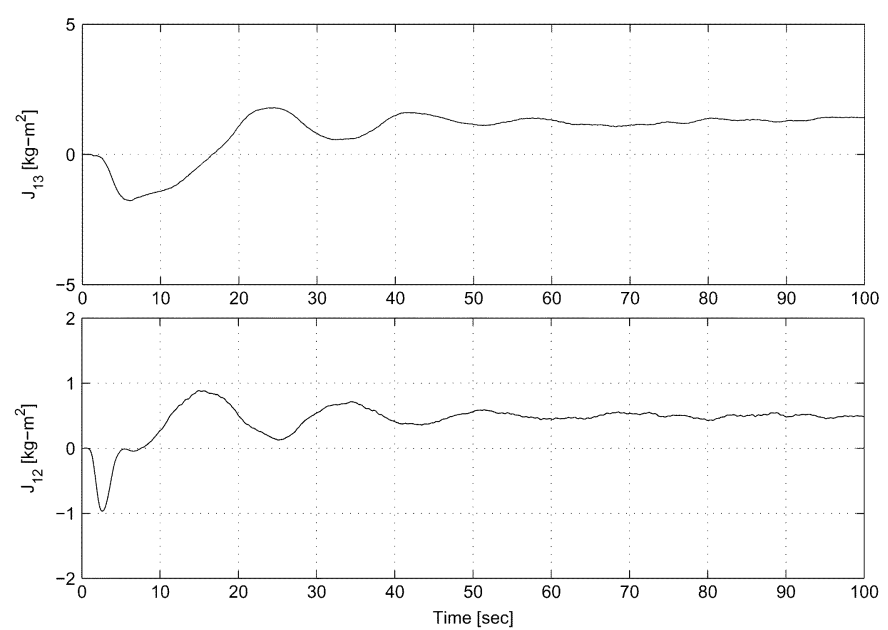

Fig. 20. Inertia estimates for $\nu_{1}=\left[\begin{array}{lll}25 & 0 & 0\end{array}\right]^{\mathrm{T}}$.

Proposition 1, we use two constant maneuvers to identify the off-diagonal entries of the inertia matrix. Thus, we command the constant signals $\nu_{1}=\left[\begin{array}{lll}25 & 0 & 0\end{array}\right]^{\mathrm{T} \%} / \mathrm{s}$ and $\nu_{2}=\left[\begin{array}{lll}0 & 25 & 0\end{array}\right]^{\mathrm{T} \%} / \mathrm{s}$. Figs. 19, 20, 21, and 22 show the response of the TACT to the reference angular velocities.
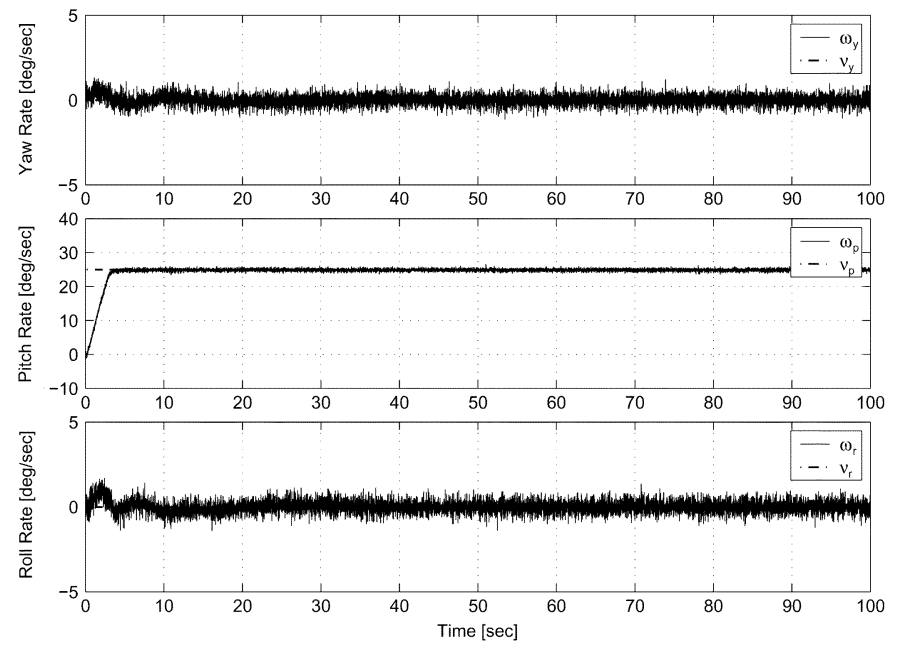

Fig. 21. Angular velocity tracking for $\nu_{2}=\left[\begin{array}{llll}0 & 25 & 0\end{array}\right]^{\mathrm{T}}$.

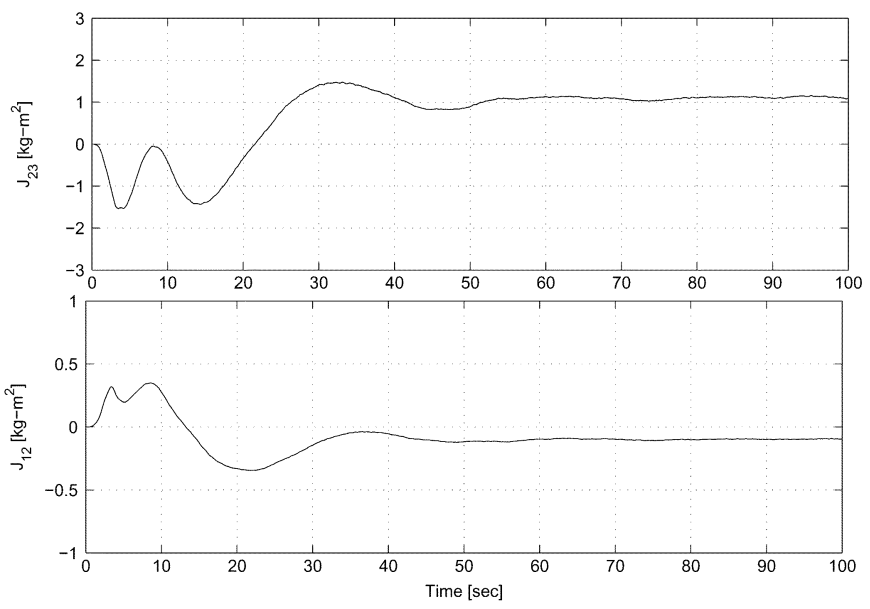

Fig. 22. Inertia estimates for $\nu_{2}=\left[\begin{array}{lll}0 & 25 & 0\end{array}\right]^{\mathrm{T}}$.

Since the TACT is constrained to a pitch angle of magnitude less than $45^{\circ}$, it is not possible to allow the TACT to follow the reference command $\nu_{2}$ starting from a position as in Fig. 10, since a pitch rate of $25 \%$ s might result in a collision with the supporting pillar. Therefore, before commanding $\nu_{2}$, TACT was rotated by $90^{\circ}$ about the $x$-axis (see Fig. 12 for an illustrative diagram of the body-fixed axes for the TACT). In the new position, the $y$-axis points vertically and, hence, a pitch maneuver (i.e., rotation about $y$-axis) involves rotations in the horizontal plane, thus, avoiding collision with the supporting pillar.

Ideally, the center of mass is balanced to lie at the pivot point of the TACT, and thus, there are no gravitational moments. Thus, experiments can be performed starting from an arbitrary initial attitude since attitude does not play a role in the dynamics of the system. However, in practice, there is some residual gravitational moment due to unbalanced mass distribution. Hence, the TACT has to be rebalanced to attain the new initial configuration. Due to this balancing requirement, the reference signals $\nu_{1}$ and $\nu_{2}$ were applied to different mass configurations. Thus, the inertia estimates for the two cases cannot be compared. 
The gains are chosen to be

$$
K=\left[\begin{array}{ccc}
14 & 0 & 0 \\
0 & 14 & 0 \\
0 & 0 & 3
\end{array}\right] \quad Q=\operatorname{diag}(70,50,50,14,14,25) .
$$

Figs. 20 and 22 show that the off-diagonal inertia estimates converge. Although the actual inertia values are not known, calculations on a simple model of the TACT suggest that the estimates obtained experimentally have the same order of magnitude as the values computed for the off-diagonal terms of the inertia matrix. To estimate the remaining entries of the inertia matrix, we need to use reference angular velocities that satisfy the conditions of Theorem 2 or the conditions mentioned in Proposition 2.

\section{CONCLUSION}

An adaptive feedback control algorithm is developed that provides global tracking of reference angular velocity signals. The control algorithm assumes no knowledge of the inertia of the body and is, thus, unconditionally robust with respect to this parametric uncertainty. Using a Lyapunov argument, it is shown that the angular velocity tracking error converges to zero and an analytical expression for an upper bound on the magnitude of the required torque is presented for a given reference signal. Furthermore, the control algorithm is used to identify the inertia matrix when the reference angular velocity signal satisfies certain conditions. Numerical simulations demonstrate tracking and identification of the inertia matrix under such conditions. Finally, implementation issues using the TACT to test the control algorithms are discussed. Results obtained from the experiments validate the effectiveness of the adaptive control algorithm in tracking an angular velocity reference and estimating the moment of inertia.

\section{REFERENCES}

[1] N. A. Chaturvedi, "Adaptive tracking of angular velocity for a planar rigid body with unknown models for inertia and input nonlinearity," IEEE Trans. Contr. Syst. Technol., vol. 14, no. 4, Jul. 2006.

[2] R. W. Brockett, "Asymptotic stability and feedback stabilization," Differential Geometric Contr. Theory, Progress in Mathematics, vol. 27, pp. 181-191, 1983.

[3] D. Aeyels and M. Szafranski, "Comments on the stabilizability of the angular velocity of a rigid body," Syst. Control Lett., vol. 10, no. 1, pp. 35-39, Jan. 1988.

[4] E. D. Sontag and H. J. Sussmann, "Further comments on the stabilizability of the angular velocity of a rigid body," Syst. Control Lett., vol. 12, no. 3, pp. 213-217, Apr. 1989.

[5] C. I. Byrnes and A. Isidori, "New results and examples in nonlinear feedback stabilization," Syst. Control Lett., vol. 12, no. 5, pp. 437-442, Jun. 1989

[6] C.-J. Wan and D. Bernstein, "Nonlinear feedback control with global stabilization," Dyn. Control, vol. 5, no. 4, pp. 321-346, Oct. 1995.

[7] H. Krishnan, N. H. McClamroch, and M. Reyhanoglu, "Attitude stabilization of a rigid spacecraft using two control torques: A nonlinear control approach based on the spacecraft attitude dynamics," Automatica, vol. 30, no. 6, pp. 1023-1027, Jun. 1994.

[8] H. Krishnan, N. H. McClamroch, and M. Reyhanoglu, "Attitude stabilization of a rigid spacecraft using two momentum wheel actuators," AIAA J. Guid. Contr. Dyn., vol. 18, no. 2, pp. 256-263, Mar--Apr. 1995.
[9] H. Krishnan, N. H. McClamroch, and M. Reyhanoglu, "Attitude stabilization of a rigid spacecraft using momentum wheel actuators operating in a failure mode," World Space Congr., Aug. 1992, IAF-92-0035.

[10] H. Krishnan, M. Reyhanoglu, and N. H. McClamroch, "Attitude stabilization of a rigid spacecraft using gas jet actuators in a failure mode," in Proc. 31st IEEE Conf. Dec. Contr., 1992, pp. 1612-1617.

[11] R. Outbib and G. Sallet, "Stabilizability of the angular velocity of a rigid body revisited," Syst. Control Lett., vol. 18, no. 2, pp. 93-98, Feb. 1992.

[12] J. L. Junkins and J. D. Turner, Optimal Spacecraft Rotational Maneuvers. New York: Elsevier, 1985.

[13] K. D. Bilimoria and B. Wie, "Time-optimal three-axis reorientation of a rigid spacecraft," J. Guid. Contr. Dyn., vol. 16, no. 3, pp. 446-452, May/Jun. 1993.

[14] B. Wie, R. Sinha, J. Sunkel, and K. Cox, "Robust fuel- and time-optimal control of uncertain flexible space structures," in Proc. Amer. Contr. Conf., 1993, pp. 2475-2479.

[15] T. A. W. Dwyer and A. L. Batten, "Exact spacecraft detumbling and reorientation maneuvers with gimbaled thrusters and reaction wheels," J. Astronaut. Sci., vol. 33, no. 2, pp. 217-232, Apr./Jun. 1983.

[16] J. Dzielski, E. Bergmann, J. Paradiso, D. Rowell, and D. Wormley, "Approach to control moment gyroscope steering using feedback linearization," J. Guid. Contr. Dyn., vol. 14, no. 1, pp. 96-106, Jan./Feb. 1991.

[17] M. S. de Queiroz, D. M. Dawson, S. P. Nagarkatti, and F. Zhang, Lyapunov-Based Control of Mechanical Systems, ser. Control Engineering Series. Cambridge, MA: Birkhäuser, 2000.

[18] J. L. Junkins, M. R. Akella, and R. D. Robinert, "Nonlinear adaptive control of spacecraft maneuvers," J. Guid. Contr. Dyn., vol. 20, no. 6, pp. 1104-1110, Nov./Dec. 1997.

[19] J. Ahmed, V. T. Coppola, and D. S. Bernstein, "Asymptotic tracking of spacecraft attitude motion with inertia matrix identification," J. Guid. Contr. Dyn., vol. 21, no. 5, pp. 684-691, Sep./Oct. 1998.

[20] H. K. Khalil, Nonlinear Systems. Upper Saddle River, NJ: PrenticeHall, 2002

[21] D. S. Bernstein, N. H. McClamroch, and A. Bloch, "Development of air-spindle and triaxial air-bearing testbed for spacecraft dynamics and control experiments," in Proc. Amer. Contr. Conf., 2001, pp. 3967-3972.

[22] S. Cho, J. Shen, N. H. McClamroch, and D. S. Bernstein, "Equations of motion of the triaxial control testbed," in Proc. 40 th IEEE Conf. Dec. Contr., 2001, pp. 3429-3434.

[23] J. Shen, A. Sanyal, N. A. Chaturvedi, D. S. Bernstein, and N. H. McClamroch, "Dynamics and control of a 3D pendulum," in Proc. 43rd IEEE Conf. Dec. Contr., 2004, pp. 323-328.

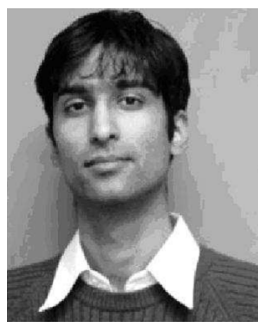

Nalin A. Chaturvedi received the B.Tech. and M.Tech. degrees in aerospace engineering from the Indian Institute of Technology, Bombay, India, in 2003, where he received the Institute Silver Medal. He is currently pursuing the Ph.D. degree in aerospace engineering at the University of Michigan, Ann Arbor.

He is a Research Fellow with the University of Michigan. His main research interests include nonlinear stability theory with applications to aerospace and mechanical systems/robotics, geometric mechanics, nonlinear and geometric control, nonlinear dynamical systems, state estimation, and adaptive control with applications to nonaffine systems.

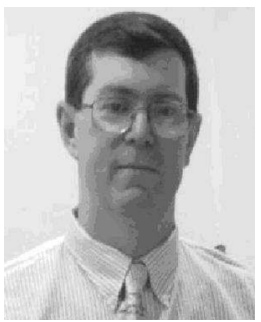

Dennis S. Bernstein (M'82-F'00) is a Professor in the Aerospace Engineering Department at the University of Michigan, Ann Arbor. His research interests include system identification, state estimation, and adaptive control, with application to vibration and flow control and data assimilation.

Dr. Bernstein is currently the Editor-in-Chief of the IEEE Control Systems Magazine. He is the author of Matrix Mathematics, Theory, Facts, and Formulas with Application to Linear Systems Theory (Princeton Univ. Press, 2005). 


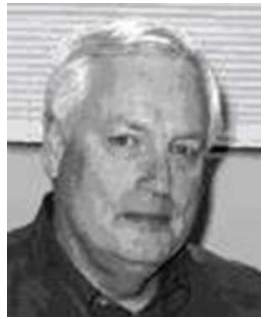

N. Harris McClamroch (M'66-F'88) received the $\mathrm{Ph} . \mathrm{D}$. degree in engineering mechanics from The University of Texas at Austin, Austin.

In 1967, he joined the University of Michigan, Ann Arbor, where he is currently a Professor in the Department of Aerospace Engineering. During the past fifteen years, his primary research interest has been in nonlinear control. He has worked on many control engineering problems arising in flexible space structures, robotics, automated manufacturing, control technologies for buildings and bridges, and

aerospace flight systems.

Dr. McClamroch received the Control Systems Society Distinguished Member Award and the IEEE Third Millennium Medal. He has served as Associate Editor and Editor of the IEEE TRANSACTIONS ON AUTOMATIC CONTROL, and he has held numerous positions, including President, in the IEEE Control Systems Society.

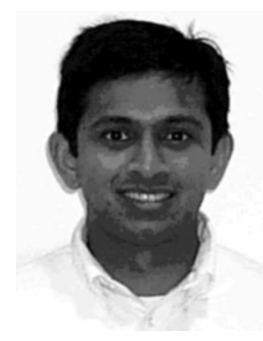

J. Ahmed received the Ph.D. degree with a focus on spacecraft control from the University of Michigan in 2000.

He is currently with the Research and Technology Center of the Robert Bosch Corporation, Palo Alto, CA. His current interests include the development of model-based control for complex physical systems involving thermal-chemical-fluid interactions.

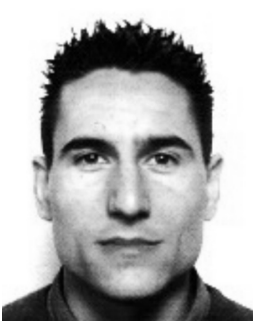

brid predictive control.
F. Bacconi was born in Florence, Italy on April 26, 1974. He received his M.S. degree cum laude in automation and control engineering from the University of Florence, Florence, Italy, in 2002. The topic of his M.S. thesis was attitude control of underactuated spacecraft in the presence of actuator failures. He is currently pursuing the $\mathrm{Ph}$.D. degree in automatic control at the Dipartimento di Sistemi e Informatica at the Università di Firenze.

His research interests include areas of control of aerospace systems, formation flying control, and hy- 